\title{
Novel approach to distinguish between vacuum UV-induced ice photodesorption and photoconversion Investigation of $\mathrm{CH}_{4}, \mathrm{CH}_{3} \mathrm{OH}$, and $\mathrm{CH}_{3} \mathrm{CN}$
}

\author{
M. Bulak ${ }^{1,2}$, D. M. Paardekooper ${ }^{3}$, G. Fedoseev ${ }^{1,2}$, and H. Linnartz ${ }^{1,2}$ \\ ${ }^{1}$ Laboratory for Astrophysics, Leiden Observatory, Leiden University, PO Box 9513, 2300 RA Leiden, The Netherlands \\ e-mail: bulak@strw.leidenuniv.nl \\ 2 Leiden Observatory, Leiden University, PO Box 9513, 2300 RA Leiden, The Netherlands \\ 3 Science Division, Jet Propulsion Laboratory, California Institute of Technology, Pasadena, CA 91109, USA
}

Received 11 December 2019 / Accepted 11 February 2020

\begin{abstract}
Context. In cold regions of the interstellar medium with intense ultraviolet radiation fields, photodesorption has been suggested as a nonthermal desorption mechanism promoting the transition of molecules from the solid state to the gas phase. Laboratory experiments measuring photodesorption rates are crucial in attempting to explain high molecular gas phase abundances of species that are expected to form in the solid state, such as methane, methanol, and acetonitrile, and to aid astrochemical modeling. Due to the convoluted competition between photodesorption and photoconversion, it is far from trivial to derive accurate photodesorption rates. Aims. The aim of this study is to apply a new methodology to discriminate between the two processes. The method has been validated using the well-studied case of $\mathrm{CO}$ and extended to $\mathrm{CH}_{4}, \mathrm{CH}_{3} \mathrm{OH}$, and $\mathrm{CH}_{3} \mathrm{CN}$.

Methods. Vacuum ultraviolet (VUV; photon energy of 7-10.2 eV) irradiated ices at $20 \mathrm{~K}$ are studied, first as a pure $\mathrm{CH}_{4}, \mathrm{CH}_{3} \mathrm{OH}$, or $\mathrm{CH}_{3} \mathrm{CN}$ ice and subsequently with an Ar coating on top. The latter is transparent to the VUV photons (wavelength below $200 \mathrm{~nm}$ ), but it quenches the photodesorption process. Comparing the laser desorption post ionization time-of-flight mass spectrometry of the ices with and without the Ar coating provides information on the different interactions of the VUV photons with the ice.

Results. The newly developed experimental technique allowed for a derivation of photodesorption rates for ices at $20 \mathrm{~K}$ of: $\mathrm{CO}(3.1 \pm 0.3) \times 10^{-3}$ mol. photon ${ }^{-1}, \mathrm{CH}_{4}(3.1 \pm 0.5) \times 10^{-2}$ mol. photon ${ }^{-1}$, and upper limits for $\mathrm{CH}_{3} \mathrm{OH}\left(<6 \times 10^{-5}\right.$ mol. photon $\left.{ }^{-1}\right)$ and $\mathrm{CH}_{3} \mathrm{CN}\left(<7.4 \times 10^{-4}\right.$ mol. photon $\left.{ }^{-1}\right)$; in the latter case, no literature values have been reported yet. The newly introduced approach provides more insight into the photodesorption process, in particular, for commonly observed complex organic molecules (COMs). Photoconversion cross sections are presented in the $7-10.2 \mathrm{eV}$ range. The possible role of photodesorption and photoconversion in the formation of interstellar COMs is discussed.
\end{abstract}

Key words. astrochemistry - ISM: molecules - ISM: clouds - ultraviolet: ISM - methods: laboratory: solid state molecular processes

\section{Introduction}

Astronomical observations show that the interstellar medium (ISM) contains many different complex organic molecules (COMs), which, by definition, are carbon bearing species made up from six or more atoms (Herbst \& van Dishoeck 2009). As their gas phase formation rates are low, it is generally accepted that COMs form on icy dust grains, as shown in a number of dedicated laboratory experiments (Gerakines et al. 1996; Watanabe \& Kouchi 2002; Fuchs et al. 2009; Fedoseev et al. 2015; Abplanalp \& Kaiser 2019). Thermal desorption efficiently releases ice species into the gas phase (Collings et al. 2004); however, COMs are also observed in interstellar regions with temperatures well below their thermal desorption temperatures, requiring alternative mechanisms to explain the observed gas phase abundances (Cernicharo et al. 2012; Guzmán et al. 2013; Vastel et al. 2014; Öberg et al. 2015). In recent decades, a number of mechanisms have been investigated in an attempt to explain the nonthermal desorption of frozen molecules. These include direct cosmic-ray-induced desorption, X-Ray-induced desorption, vacuum ultraviolet (VUV) photodesorption, reactive desorption, and different types of codesorption (Dartois et al. 2015; Dupuy et al. 2017, 2018; Chuang et al. 2018; Ligterink et al. 2018; Oba et al. 2018).

In particular, VUV photodesorption is considered to be an effective mechanism with regard to $\mathrm{CO}$ ice high rates on the order of $10^{-3}$ to a few times $10^{-2}$ mol. photon ${ }^{-1}$, which were determined in the laboratory (for an overview see Fig. 5 in Paardekooper et al. 2016a,b and references therein). As a result, state-of-the-art astrochemical gas-grain models include photodesorption rates based on laboratory works (Garrod \& Pauly 2011; Walsh et al. 2014; Kalvāns 2015). However, the number of molecules studied in laboratory experiments is limited. Models commonly use a rate of $10^{-3}$ mol. photon ${ }^{-1}$ when no value has been reported.

A major challenge in an experimental ice VUV photolysis study boils down to separating multiple phenomena that can happen within the ice upon photon absorption. Figure 1 is a simplified summary of feasible processes in solid state species on a grain surface in cold regions in the ISM or in 
laboratory experiments on a gold-coated copper substrate. A molecule embedded in the ice matrix is electronically excited by an absorbed photon (1). Upon relaxation back to the ground state (2), the molecule can transfer its energy to neighboring species, followed by the neighbor's desorption (3a, indirect desorption as in Bertin et al. 2013; van Hemert et al. 2015). Alternatively, the excited species can directly photodesorb (3b) or photodissociate (4). The latter process can be followed by dissociative photodesorption ( $3 \mathrm{c}$, transfer of excited radicals into the gas phase demonstrated in Muñoz Caro et al. 2010; Fillion et al. 2014), the "kick-out" of a fragment or parent species by an excited radical (3d, e.g., kick-out of $\mathrm{H}_{2} \mathrm{O}$ or $\mathrm{OH}$ by an energetic $\mathrm{H}$ atom as in Andersson \& van Dishoeck 2008), or product formation (5). If the newly formed species are in an excited state, they either desorb ( $3 \mathrm{e}$ - reactive photodesorption and photochemidesorption as in Martín-Doménech et al. 2016 and Andersson \& van Dishoeck 2008) or dissipate their energy and remain in the ice (6). The photodissociation branch is typically accessible when a molecule is excited by a photon that carries more energy than the bond dissociation energy (BDE). In that case, chemical bonds can be broken, which is the origin of radical species. The radicals at $20 \mathrm{~K}$ have a limited mobility; however, for small fragments, such as $\mathrm{H}$ or $\mathrm{OH}$, diffusion across a few layers of ice may occur (Andersson \& van Dishoeck 2008). Alternatively, if radicals are near each other, they react with little or no barrier to form larger molecules. The desorption processes described above (3a, 3b, 3c, 3d) are expected to occur for molecules in the surface layers of the ice. It has been shown that for CO ice, only the top $3 \mathrm{ML}\left(1 \mathrm{ML}=10^{15}\right.$ molecules $\left.\mathrm{cm}^{-2}\right)$ can be photodesorbed, which are considered surface layers in this work (Bertin et al. 2012). Besides photodesorption, a decrease in the abundance of the parent species is caused by photoconversion. Here, the photoconversion rate is characterized by a combination of the following two solid state processes: photodissociation, followed by recombination reactions into species other than the parent molecule, that is, photoproducts, and reactions of nondissociated, photoexcited molecules, with neighboring neutral species, which also lead to the production of other species. The focus in this work is on $\mathrm{CO}, \mathrm{CH}_{4}, \mathrm{CH}_{3} \mathrm{OH}$, and $\mathrm{CH}_{3} \mathrm{CN}$.

In order to simulate interstellar radiation fields in a laboratory setting, we used a light source with the photon energy range 7-10.2 eV. In this range, $\mathrm{CO}$, the second most abundant molecule in the ISM, does not dissociate (BDE of CO is $11.16 \mathrm{eV}$ from Kalescky et al. 2013); therefore, it is a relevant and an "easy" case to study. During CO ice photoprocessing, the decrease in an abundance of $\mathrm{CO}$ can be linked to intact photodesorption. For this reason and the fact that $\mathrm{CO}$ is an abundant constituent of the interstellar ices, $\mathrm{CO}$ photodesorption has been studied in much detail by several research groups. The first experiments monitored the initial amount and subsequent loss of $\mathrm{CO}$ ice (as a function of VUV fluence) using reflection-absorption infrared spectroscopy (RAIRS). During the same experiments, a quadrupole mass spectrometer (QMS) signal of $\mathrm{m} / \mathrm{z}=28 \mathrm{can}$ be recorded, which allows for the calibration of the gas phase QMS readings to the decline of the abundance of solid state CO detected by infrared (IR) spectroscopy (Öberg et al. 2009a; Fayolle et al. 2011).

A limitation of the IR-based method is that similar vibrational modes of different species may overlap. This is particularly true for COMs. In addition, unknown (temperature and environment dependent) IR band strengths add uncertainty to quantifying results. For molecules with a BDE that is lower than $10.2 \mathrm{eV}$, it is challenging to disentangle the effects of dissociation and desorption since both these processes simultaneously

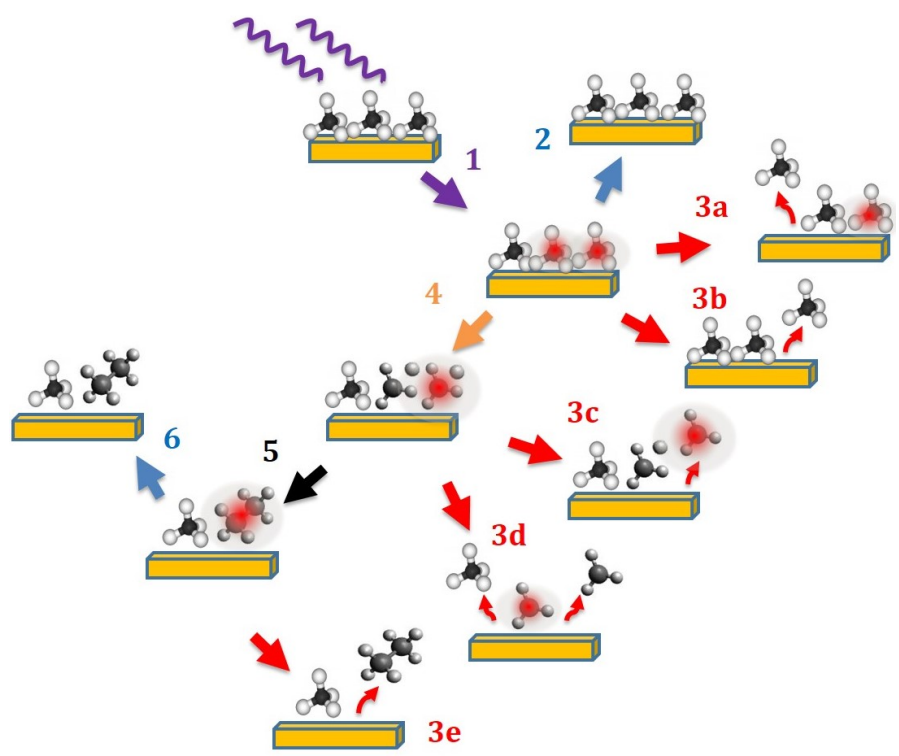

Fig. 1. Competition between processes taking place in solid state species upon an absorption of a photon. Photodesorption processes (in red) are only possible for the top layers of the ice. All other processes can happen in both the ice bulk and surface. The schematic applies to ices at a temperature excluding thermal desorption, typically $\leq 20 \mathrm{~K}$.

deplete the parent species. In addition, RAIRS cannot be applied to molecules without IR active modes.

A QMS can be used for a gas phase analysis of desorbed species. In order to quantify results using this method, a mathematical conversion is necessary to correlate the gas phase QMS signal of a molecule (e.g., methane or methanol) to the previously measured gas phase $\mathrm{CO}$ signal (i.e., calibrated with solid state $\mathrm{C} \equiv \mathrm{O}$ stretching mode in the IR). Factors that need to be considered for this conversion are the setup and the molecule specific QMS detector sensitivity, varying pumping speeds for different molecules, electron ionization cross-sections, and fragmentation patterns. For a discussion on the calibration see Zhen \& Linnartz (2014).

Methane has been identified as a component of ice mantles toward multiple sources (e.g., Young Stellar Objects) in abundances ranging from 1 to $10 \%$ with respect to $\mathrm{H}_{2} \mathrm{O}$ (Boogert et al. 2015). The detection has motivated multiple experimental studies, which show that, in contrast to $\mathrm{CO}$, methane can be dissociated by impacting photons with an energy below $10.2 \mathrm{eV}$ $\left(\mathrm{H}-\mathrm{CH}_{3}\right.$ bond energy of $4.5 \mathrm{eV}$ from Ruscic 2015). Hence, methane photolysis is an example of the competition between photodesorption and photodissociation followed by an increasing chemical complexity. Pure ice photolysis results in various products including $\mathrm{C}_{2} \mathrm{H}_{2}, \mathrm{C}_{2} \mathrm{H}_{4}, \mathrm{C}_{2} \mathrm{H}_{6}$, and larger hydrocarbons (Gerakines et al. 1996; Paardekooper et al. 2014; Lo et al. 2015). The dissociation results in effective branching ratios of $\mathrm{CH}_{3}: \mathrm{CH}_{2}: \mathrm{CH}$ equal to $95: 4: 2$ (Bossa et al. 2015), which is quite different from similar studies performed in the gas phase (Gans et al. 2013). Intact $\mathrm{CH}_{4}$ photodesorption at $12 \mathrm{~K}$ was investigated recently in a wavelength-dependent study at the SOLEIL synchrotron (Dupuy et al. 2017). This work resulted in an intact photodesorption rate of $2.2 \times 10^{-3}$ mol. photon $^{-1}$ in a photon energy range of $9.1-13.6 \mathrm{eV}$. The photodesorption yield in that range follows the trend of the absorption spectrum of solid state methane. To date, this is the only published measurement of intact methane photodesorption. In 2016, reactive photodesorption of $\mathrm{H}_{2} \mathrm{CO}$ from a photoprocessed $\mathrm{CH}_{4}: \mathrm{H}_{2} \mathrm{O}$ 
mixture was demonstrated, whereas intact photodesorption was not (Martín-Doménech et al. 2016), which suggests that multiple desorption mechanisms play a role in understanding the process as a whole.

Solid state methanol has been detected as a significant component of the ice mantles of interstellar dust grains with abundances varying between $3 \%$ and $12 \%$ with respect to water in dense clouds (Chiar et al. 1996; Boogert et al. 2011). VUV processing of methanol ice yields more complex organics, including methyl formate, glycolaldehyde, ethylene glycol, and many others, thus making methanol one of the pillars of the interstellar chemical network (Gerakines et al. 1996; Öberg et al. 2009b; Boamah et al. 2014; Henderson \& Gudipati 2015; Maity et al. 2014; Paardekooper et al. 2016a; Chuang et al. 2017). Öberg and coworkers in 2009 were the first to study the photodesorption of methanol. A partial decrease in the IR signature was assigned to intact photodesorption and a rate of $(2.1 \pm 1.0) \times$ $10^{-3}$ mol. photon ${ }^{-1}$ was derived (Öberg et al. 2009b). Photodesorption and photodissocation were fit separately by explicitly assuming that photodesorption is a 0th order surface and photodissociation is mainly a 1 st order bulk process. In 2016, a study by Cruz-Diaz et al. determined an upper limit for intact methanol desorption to be in the $10^{-5}$ mol. photon ${ }^{-1}$ range. Simultaneously, Bertin et al. (2016) measured photodesorption of methanol in a wavelength-dependent study, using the VUV DESIRS beamline at the SOLEIL synchrotron facility. The average photodesorption rate derived for the ultraviolet (UV) interstellar radiation field profile (Mathis et al. 1983) was (1.2 \pm $0.6) \times 10^{-5}$ mol. photon ${ }^{-1}$. The measured photodesorption was proposed to result from a recombination reaction of the $\mathrm{CH}_{3} \mathrm{O}$ or $\mathrm{CH}_{2} \mathrm{OH}$ radical with a hydrogen atom. The same study also demonstrated the dissociative photodesorption of $\mathrm{CH}_{3}, \mathrm{OH}$, and $\mathrm{H}_{2} \mathrm{CO}$ at similar efficiencies to intact photodesorption. Both laboratory studies of Bertin et al. (2016) and Cruz-Diaz et al. (2016), find lower photodesorption rates of methanol by 2-3 orders of magnitude compared to previous work. When the updated laboratory photodesorption rates were applied to gas grain astrochemical models of protoplanetary disks, it was concluded that photodesorption alone is insufficient in explaining the observed gas phase methanol abundances and that other mechanisms have to be invoked (Ligterink et al. 2018).

Methyl cyanide, or acetonitrile $\left(\mathrm{CH}_{3} \mathrm{CN}\right)$, has been detected within the Solar System: in Titan's atmosphere (Cordiner et al. 2015), in comet 67P/Churyumov-Gerasimenko (Goesmann et al. 2015), and in cometary comae of Hale-Bopp (Woodney et al. 2002). In addition, there were multiple detections toward sources outside of the Solar System, including molecular clouds Sgr A and Sgr B (Solomon et al. 1971), protoplanetary disks around T Tauri stars (Bergner et al. 2018), and high and low-mass protostars (Purcell et al. 2006; Calcutt et al. 2018). $\mathrm{CH}_{3} \mathrm{CN}$ has also been recently detected to be a part of the icy mantle in a circumstellar disk around V883 Ori. The detection was possible due to a shifted sublimation front, which was caused by an outburst of a young star (Lee et al. 2019).

Acetonitrile $\left(\mathrm{CH}_{3} \mathrm{CN}\right)$ plays an important role in the nitrilebased chemistry. With available solid state formation routes (Garrod et al. 2008) and a high thermal desorption temperature ( $140 \mathrm{~K}$ in laboratory conditions), energetic processing of $\mathrm{CH}_{3} \mathrm{CN}$ ice becomes an important subject. It has been investigated in a number of largely qualitative studies (Hudson \& Moore 2004; Abdulgalil et al. 2013). Final products of VUV photolysis of pure acetonitrile have been identified to be as follows: $\mathrm{HCN}, \mathrm{CH}_{4},\left(\mathrm{CH}_{2} \mathrm{CN}\right)_{2}$, and isomerization to $\mathrm{CH}_{3} \mathrm{NC}$ and $\mathrm{H}_{2} \mathrm{CCNH}$ (Hudson \& Moore 2004; Hudson et al. 2008). Ion- irradiation and hydrolysis of $\mathrm{CH}_{3} \mathrm{CN}: \mathrm{H}_{2} \mathrm{O}$ mixtures yield a variety of amino acids (Hudson et al. 2008), which supports $\mathrm{CH}_{3} \mathrm{CN}$ as an important element of the N-bearing chemical network. A quantitative photodesorption study of acetonitrile ice is currently lacking. This is due to experimental challenges associated with disentangling VUV-induced solid state processes. In this work, we address these challenges by applying our new measurement method.

\section{Experimental}

In order to provide more insight into the photon-driven competition in the solid state, we developed a new method to measure photodesorption rates of COMs. VUV irradiated ices at $20 \mathrm{~K}$ are first studied as a pure ice species and subsequently with a protecting argon (Ar) coating on top. The latter quenches photodesorption, which becomes clear when comparing the time-of-flight mass spectra of the probed (un)coated ices. The following subsections provide information on the experimental setup, measurement principle, and applied methodology.

\subsection{Setup overview}

The experiments were carried out in an ultra-high vacuum (UHV) apparatus MATRI ${ }^{2}$ CES (Mass Analytical Tool for Reactions in Interstellar ICES). A thorough description of the system is given by Paardekooper et al. (2014).

The vacuum system consists of two parts: a main chamber and a time-of-flight chamber, which are connected with each other by a gate valve. Both are evacuated by turbomolecular pumps, which are supported by a prepump. The base pressure in the chambers, measured by inverted magnetron gauges, is in the $10^{-10}$ mbar range. The pressure is comparable to a density of $10^{7} \mathrm{H}_{2}$ particles $\mathrm{cm}^{-3}$ which, combined with low temperature, make for a reasonable representation of a dense cloud core. In the main chamber, a gold-coated copper block serves as a nonreactive substrate onto which the ices are grown. It is in thermal contact with a closed-cycle helium cryostat, which allows for surface temperatures as low as $20 \mathrm{~K}$. The temperature of the substrate is controlled by a thermocouple and a resistive heater connected to a temperature regulator. The available temperature range is between 20 and $300 \mathrm{~K}$, and it is set with an absolute precision of less than $1 \mathrm{~K}$. The cryostat is mounted on a two-dimensional translation stage. A stepper motor allows for automized control of the position of the substrate and of the ices that are grown onto it in the vertical direction (z-axis). In the horizontal direction ( $y$-axis), manual control is supported by a translation stage.

Ices are formed on the substrate via front deposition, which is controlled by a calibrated high precision leak valve. The gas mixing line is evacuated by a turbomolecular pump that is supported by a scroll pump, which allows pressures in the $10^{-4} \mathrm{mbar}$ range. Samples in both the gas phase and liquid phase can be introduced to the main chamber. In the experiments, we used $\mathrm{CO}$ (Linde, 99.997\%), $\mathrm{CH}_{4}$ (Praxair, 99.999\%), $\mathrm{CH}_{3} \mathrm{OH}$ (SigmaAldrich, Chromasolv, 99.99\%), $\mathrm{CH}_{3} \mathrm{CN}$ (VWR, <10 ppm of water), $\mathrm{CD}_{3} \mathrm{CN}$ (Sigma-Aldrich, $\geq 99.8 \% \mathrm{D}$ ), and Ar (Linde, $\geq 99.999 \%$ Ar). To prevent contamination from the air, all liquid samples were taken through two freeze-pump-thaw cycles prior to deposition. The front deposition proceeds at an angle of $85^{\circ}$ with respect to the substrate's surface plane.

The spectral energy distribution (SED) of the interstellar radiation field includes Lyman- $\alpha(121 \mathrm{~nm})$, a molecular $\mathrm{H}_{2}$ emission continuum (130-165 $\mathrm{nm}$ ), and can be closely simulated by 
a microwave discharge hydrogen lamp (MDHL). To accurately determine the photoprocessing rates, it is crucial to calibrate the wavelength dependent flux of the light source. The SED of the lamp is strongly dependent on its operational conditions (Ligterink et al. 2015). We used an $\mathrm{H}_{2}$ pressure of 1.44 mbar and $80 \mathrm{~W}$ of microwave power, which results in an SED, which is shown in (Fig. 4 in Paardekooper et al. 2016a,b). The lamp is attached to the chamber via a $\mathrm{MgF}_{2} \mathrm{UHV}$ viewport, which directly faces the substrate. To measure the emitted photon flux, a calibrated silicone photodiode was placed at the position of the substrate (following the approach in Paardekooper et al. 2016a,b). The measured photocurrent includes contributions from photons in both VUV and visible ranges. In order to specifically determine the VUV flux, at the same plasma conditions, we operated a sealed lamp with a glass seal inserted between the lamp and $\mathrm{MgF}_{2}$ window, which absorbs photons at $\lambda<300 \mathrm{~nm}$ and transmits at $90 \%$ efficiency in the visible range. The difference in the photocurrent $(\mathrm{i}(\lambda))$ between the two lamp settings was used to calculate the VUV photon flux contribution. The flux, $\mathrm{F}(\lambda)$, was determined to be $(2.5 \pm 0.5) \times 10^{14}$ photons $\mathrm{cm}^{-2} \mathrm{~s}^{-1}$, using the equation below:

$F(\lambda)=\frac{i(\lambda)}{e \cdot \epsilon(\lambda)}$

It takes the quantum efficiency of the photodiode, $\epsilon(\lambda)$, and the elementary charge $\left(e=1.602 \times 10^{-19} \mathrm{C}\right)$ into account.

Probing the photoprocessed ice is done with laser desorption post-ionization time-of-flight mass spectrometry (LDPI TOFMS). It should be noted that this laser-induced desorption is, in fact, nothing more than a direct ablation process, which is not related in any way with the VUV-induced processes we want to study here. In the following, we use "ablation" to avoid confusion. For ablation, a Nd:YAG laser's third harmonic frequency is used. The 3-4 ns pulses at $355 \mathrm{~nm}$ carry up to 3-11 $\mathrm{mJ}$ per pulse (molecule-dependent) at $5 \mathrm{~Hz}$. The unfocused beam is trimmed to a diameter of $1 \mathrm{~mm}$ and pointed toward the substrate at an angle of $30^{\circ}$. Synchronization of the vertical motion of the substrate and laser shots allows one to probe a "fresh" spot with every laser shot. Each shot desorbs a plume of material that floats in the direction orthogonal to an ionizing sheet of electrons $(70 \mathrm{eV})$. After an optimized time delay, generated cations are extracted from the main chamber into the field-free time-offlight tube. The ions are reflected using a reflectron at the end of the flight tube. The reflectron increases the separation between ions with a different $\mathrm{m} / \mathrm{z}$ and minimizes the initial velocity distribution within the desorption plume. After reflection, the ions pass through the field-free tube again and are detected with a $40 \mathrm{~mm}$ micro-channel plate (MCP) detector. The signal from the detector is recorded via a data acquisition card (DAQ) as the voltage versus time delay, which can be converted to a mass over charge ratio. Timing of the laser shots, ion extraction, and data acquisition is set through a delay generator and is controlled via a custom-made Labview routine. Typically 85 TOF spectra, which correspond to fresh spots along one column on the substrate, are collected and averaged to one mass spectrum. Afterwards, the substrate can be manually moved to a different $y$-axis position and the measurement can be repeated to probe the effects for further increasing VUV fluence.

\subsection{Thickness measurements}

The ice deposition procedure has been calibrated using a series of measurements with an intensity stabilized He-Ne laser (Hudgins et al. 1993). For each ice growth, the gas pressure in the mixing line, needle valve setting, and temperature are recorded. During the deposition, a photodiode records a laser interference pattern as the laser beam is reflected from the ice surface and the substrate. The equation expressing the ice thickness (d) as a function of the number of interference fringes $(m)$ is given by:

$d=\frac{m \cdot \lambda}{2 \cdot \frac{n_{1}}{n_{0}} \cdot \cos \theta}$,

where $\lambda$ is the wavelength of the He-Ne laser $(632.8 \mathrm{~nm}), n_{0}$ and $n_{1}$ are refractive indices of the vacuum and ice, and $\theta$ is the angle of refraction $\left(3^{\circ}\right)$. The growth rate $\left(\tau\right.$, in mol. $\left.\mathrm{cm}^{-2} \mathrm{~s}^{-1}\right)$ can be determined from:

$\tau=\frac{d \cdot \rho \cdot N_{\mathrm{A}}}{M \cdot t}$

where $\mathrm{t}$ is the time corresponding to the thickness $d$ (calculated in Eq. (2)), $N_{\mathrm{A}}$ is Avogadro's number $\left(6.022 \times 10^{23} \mathrm{~mole}^{-1}\right)$, $\rho$ is the ice density in $\left(\mathrm{g} \mathrm{cm}^{-3}\right)$, and $M$ is the molar mass of the species (in $\mathrm{g} \mathrm{mole}^{-1}$ ). The ice thickness (in mol. $\mathrm{cm}^{-2}$ ) has the same units as column density and is controlled by changing the deposition time while keeping all of the other parameters constant. We performed these measurements for $\mathrm{Ar}, \mathrm{CO}, \mathrm{CH}_{4}$, $\mathrm{CH}_{3} \mathrm{OH}$, and $\mathrm{CH}_{3} \mathrm{CN}$. Respective densities and refractive indices used to calculate the growth rates are: $1.76 \mathrm{~g} \mathrm{~cm}^{-3}$ (Dobbs et al. 1956) and 1.29 (Sinnock \& Smith 1968); $0.8 \mathrm{~g} \mathrm{~cm}^{-3}$ and 1.27 (Roux et al. 1980); $0.4 \mathrm{~g} \mathrm{~cm}^{-3}$ and 1.329 (Brunetto et al. 2008); and $0.64 \mathrm{~g} \mathrm{~cm}^{-3}$ (Luna et al. 2018) and 1.33 (Weast 1972), $0.8 \mathrm{~g} \mathrm{~cm}^{-3}$, and 1.34 (assumed to be the same as for liquid at room temperature Riddick et al. 1986). The deposition method allows one to reproduce ices with the thickness varying within $5-10 \%$. This was concluded after a comparison of time-of-flight signals probing nonoverlapping spots across the substrate surface.

The laser interference calibration method is limited to ices for which refractive indices are known (pure species). This might change as a result of a recently introduced new broad band technique, which holds the potential to also derive such parameters for mixed ices (Kofman et al. 2019).

\subsection{Experimental procedure}

A series of two structurally different experiments were performed for each of the ices. The first experiment probed the effects of VUV photoprocessing of an ice at $20 \mathrm{~K}$ (photodissociation, photodesorption, radical recombination). For the second experiment, an additional layer of Ar (around 50 ML) was deposited on top of the ice of interest, while the same procedure was utilized to probe the VUV photoprocessing of the ice. Here, it should be noted that given the large volume of the sample holder, $20 \mathrm{~K}$ is the lowest temperature that is achievable. This temperature is several Kelvin higher than the typical $12-15 \mathrm{~K}$ values used in previous ice experiments, but it is still well below the accretion onset of $\mathrm{CO}$ and other astronomically relevant species in the ISM. The extra Ar layer is added to fully quench the photodesorption process; surface species, typically restricted to the upper few monolayers, are prohibited from leaving the surface; photodissociation, however, can still take place, like in the original ice without Ar coating. At $20 \mathrm{~K}$, diffusion of molecules or an energy transfer between more than a few monolayers is negligible (Cuppen \& Herbst 2007). Therefore in experiments with the Ar layer, intact, dissociative, and reactive photodesorption (being surface or subsurface processes) are effectively quenched. Ar, as a noble gas, does not react with our 
species of interest. Moreover, it does not absorb light in the VUV range (Schnepp \& Dressler 1960), which allows for a comparison of coated and uncoated experiments (same flux). The direct consequence of this approach is that a difference between the (un)coated ices can be directly linked to a difference that originates from photodesorption and photoconversion compared to photoconversion only.

To understand the principle concept of the method applied here, it is important to realize that we recorded all species remaining in the ice rather than selected species leaving the ice. The resulting mass spectra include contributions from molecular ion peak signals, respectively: $m / z=28,16,32,41$ for $\mathrm{CO}$, $\mathrm{CH}_{4}, \mathrm{CH}_{3} \mathrm{OH}$, and $\mathrm{CH}_{3} \mathrm{CN}$, as well as molecule-specific fragmentation patterns that arise due to the used electron ionization method.

A previous photodesorption study on $\mathrm{CO}$ ice using $\mathrm{MATRI}^{2} \mathrm{CES}$ demonstrates a linear relation between the integrated signal from a laser-desorbed plume, which was collected and summed up over multiple extraction timings, and the surface coverage and column density in the ice (Paardekooper et al. 2016b). In other words, the intensity of a signal in the MCP detector is linearly dependent on the abundance of the species in the ice. Given the initial column density of the molecule in the ice (see Sect. 2.2), it is possible to monitor its decrease as a function of VUV fluence by recording mass spectra as a function of irradiation time. This is confirmed for methane for a constant extraction time; this can be seen in Fig. 2, which shows the integrated molecular ion signal at $m / z=16$ as a function of ice thickness as well as the characteristic fragmentation pattern resulting from the electron impact ionization. The linear dependence of the signal is demonstrated for ices of up to $150 \mathrm{ML}$. In our photodesorption experiments, the maximum ice thickness amounts to $105 \mathrm{ML}$, that is, in a range where the signals can be used to determine the methane column density. This procedure along with well-calibrated deposition rates, allows for the recording of absolute column density of the species throughout experiments.

We recorded the LDPI TOF-MS spectra of pure ices for all of the species under investigation. These spectra directly correspond to the fragmentation pattern of the molecules as available on the NIST database. During our analysis, the fractional contribution of fragments of the parent species were subtracted from their respective $\mathrm{m} / \mathrm{z}$ signals and added to the molecular ion signal. By using this method, we were able to trace the fragments of photoproducts (along the same channels as the fragments of the parent species), which were being formed during the experiments. It should be noted that the fragmentation pattern of any photoproduct does not overlap with the molecular ion peak of the parent and, therefore, it does not complicate the analysis.

There is a fundamental limitation to photolysis experiments. Ideally, an experiment would entail thin ices and long irradiation times, which allow for spread-out data that expose the subtle, surface photodesorption process. However, when photodissociation is an available channel, the parent species deplete rapidly, which increases the difficulty of tracing subtle photodesorption processes. For each species, we used a different initial ice thickness and total photon fluence. We ensured that all ices were at least $5 \mathrm{ML}$ or thicker during the full experiment; as for thinner ices, the photodesorption may run into a nonlinear regime (Muñoz Caro et al. 2010; Fayolle et al. 2011). The particular thicknesses for methane and methanol experiments ( 55 and $40 \mathrm{ML}$ ) were motivated by previous studies performed on the setup with the aim to compare our results against previously published work (Bossa et al. 2015; Paardekooper et al.

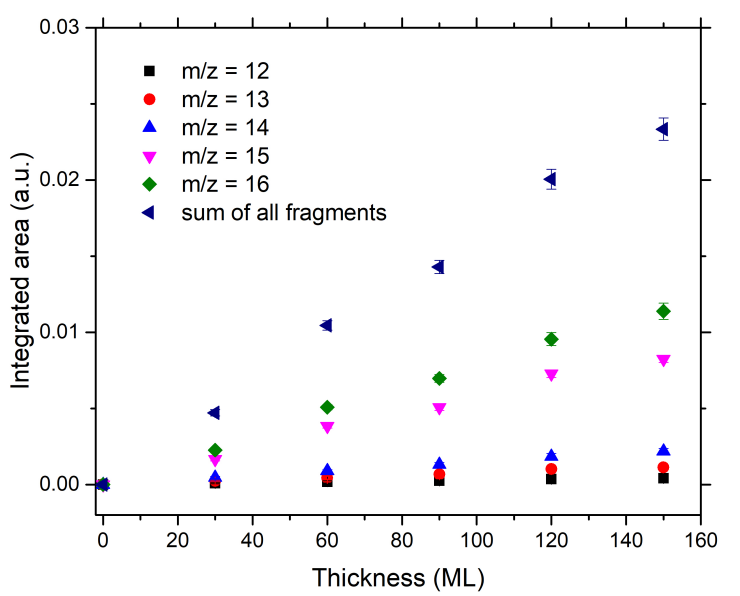

Fig. 2. Linear dependence between the thickness of the ice and intensity of methane mass peaks, arising from the electron ionization. Thickness is given in monolayers $\left(1 \mathrm{ML}=10^{15}\right.$ molecules $\left.\mathrm{cm}^{-2}\right)$.

2016a). In case of acetonitrile (8 ML), we chose to deposit a thin ice to magnify the surface versus the bulk processes. A drawback specific to our method is that the Ar cap, despite being inert, may increase the efficiency of chemistry on the ice surface. Quenching the photodesorption of radicals, allows for a higher probability that their recombination forms bigger species in the ice (not necessarily back into the parent), also known as the "cage effect". Previous studies show an efficiency of dissociative photodesorption for methanol to be between $10^{-5}$ and $10^{-6}$ mol. photon ${ }^{-1}$ (Bertin et al. 2016). However, only a fraction of these photodesorbed fragments would have recombined into the parent and therefore influenced the rates derived here. The efficiency of this process is likely to be molecule dependent and in this work it is assumed to be below the detection levels.

Associated with our method of probing the ice by laser ablation is also a possibility of thermally triggered chemistry. That effect can only be seen when using full laser power for the capped ices. To prevent that, the laser power was tuned to the minimum, thus still allowing a good time-of-flight signal.

\subsection{Analysis methods}

We derived the photodesorption rates by fitting a linear function to the difference between Ar coated and uncoated ice photolysis experiments. The next analysis step is applicable to molecules that photodissociate within the used photon energy range: methane, methanol, and acetonitrile. When an Ar layer quenches photodesorption, a decrease in the abundance of the parent species is caused by photoconversion. A photoconversion cross section can be derived by fitting the coated experimental data with an exponential function:

$N(t)=N_{0} \mathrm{e}^{-\phi t \sigma_{\text {photoconv }}}$,

where $N(t)$ and $N_{0}$ are time dependent and initial column densities in mol. $\mathrm{cm}^{-2}, \phi$ is flux in photons $\mathrm{cm}^{-2} \mathrm{~s}^{-1}, \mathrm{t}$ is the irradiation time in seconds, and $\sigma_{\text {photoconv }}$ is the photoconversion cross section in $\mathrm{cm}^{2}$.

Under low VUV fluence, when the recombination reactions in the ice are not very significant, photoconversion following an exponential decay can be approximated by a linear fit. In that case, the slope of the fit yields photoconverted mol. photon ${ }^{-1}$.

In order to better compare our results to different experimental conditions from other studies, all derived rates (per incident 


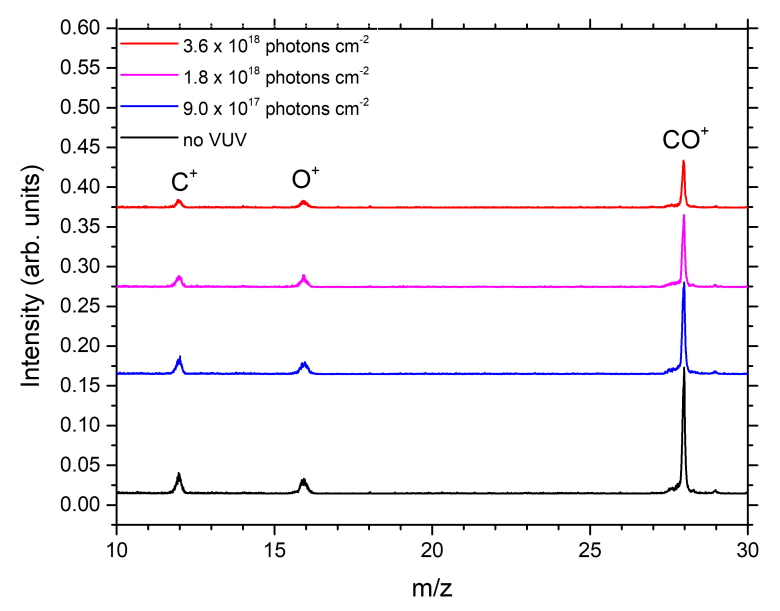

Fig. 3. LDPI TOF mass spectra of CO ice acquired at different VUV fluences. An offset between mass spectra has been inserted for clarity as is the case for other figures in this work.

photon) can be converted to effective yields (per absorbed photon). This is discussed in more detail in Sect. 4.2. This way, experiments with ices of a different thickness and with a different photon flux can be compared. When the intensity of the incident VUV photons, $I_{\text {inc }}$, crosses the ice, the light intensity $(I)$ follows the Beer-Lambert absorption law:

$I=I_{\text {inc }} \mathrm{e}^{-\sigma(\lambda)_{\text {abs }} N}$

where $I_{\text {inc }}$ is in photons $\mathrm{cm}^{-2} \mathrm{~s}^{-1}$ (see Sect. 2.1), $\sigma_{\text {abs }}$ is the average absorption cross-section (in $\mathrm{cm}^{2}$, taken from the literature), and $N$ is the column density in mol. $\mathrm{cm}^{-2}$. By applying the absorption cross section of species from the literature (an average value for the wavelength range 120-165 nm), we can determine how many photons are absorbed by a specific ice column density. For example, for CO (see the next subsection), the absorption cross section is $4.7 \times 10^{-18} \mathrm{~cm}^{2}$ and the top $3 \mathrm{ML}$ absorb $\sim 1.5 \%$ of photons, while the whole ice, $21 \mathrm{ML}$ absorb $10 \%$ of incident photons (Cruz-Diaz et al. 2014a).

\subsection{Proof of concept with $\mathrm{CO}$}

Carbon monoxide is one of the most abundant constituents of the ice mantles on interstellar dust grains, yet, it is also detected in the gas phase in regions where the temperature is below its freeze-out (Willacy \& Langer 2000). That motivated detailed experimental studies of photodesorption of CO (Öberg et al. 2009a; Muñoz Caro et al. 2010; Fayolle et al. 2011; Chen et al. 2013; Paardekooper et al. 2016b). It makes a good case study because the $\mathrm{BDE}$ of the $\mathrm{C} \equiv \mathrm{O}$ bond, $11.16 \mathrm{eV}$, excludes photodissociation in our experiments (Kalescky et al. 2013). The $\mathrm{CO}$ ionization potential is well above the used energies. The photodesorption rates across previous studies are consistent and range from $10^{-3}$ to a few times $10^{-2}$ mol. photon ${ }^{-1}$. The differences are most likely associated with varying experimental conditions (deposition and sampling temperature, spectral energy distribution, and flux of the used VUV lamps).

Two distinct experiments have been performed on $\mathrm{CO}$ ice at $20 \mathrm{~K}$. In the first experiment, a pure layer of $\mathrm{CO}$ ice (thickness $=21 \mathrm{ML}$ ) was irradiated with VUV photons and LDPI TOF mass spectra were recorded at different irradiation times. During the second experiment, an additional Ar layer of about 50 ML was deposited on top of the $21 \mathrm{ML}$ of CO. In both experiments,

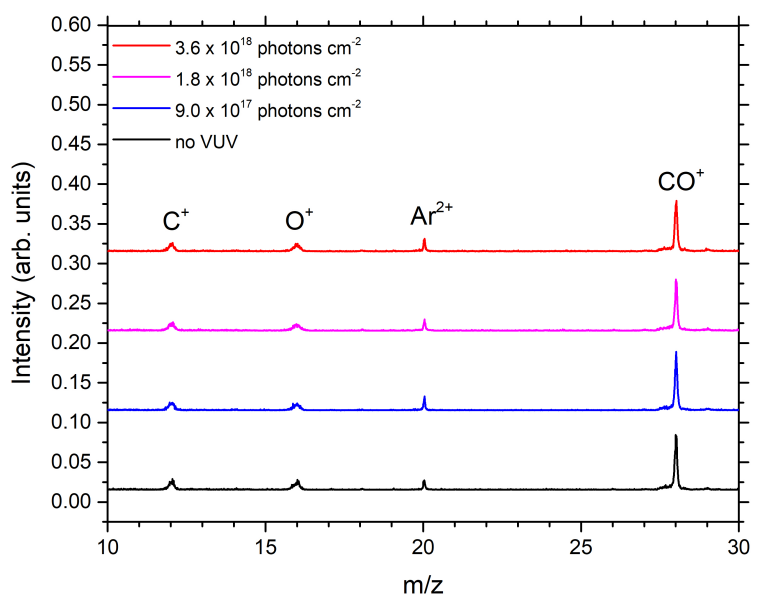

Fig. 4. LDPI TOF mass spectra of $\mathrm{CO}$ with Ar coating acquired at different VUV fluences.

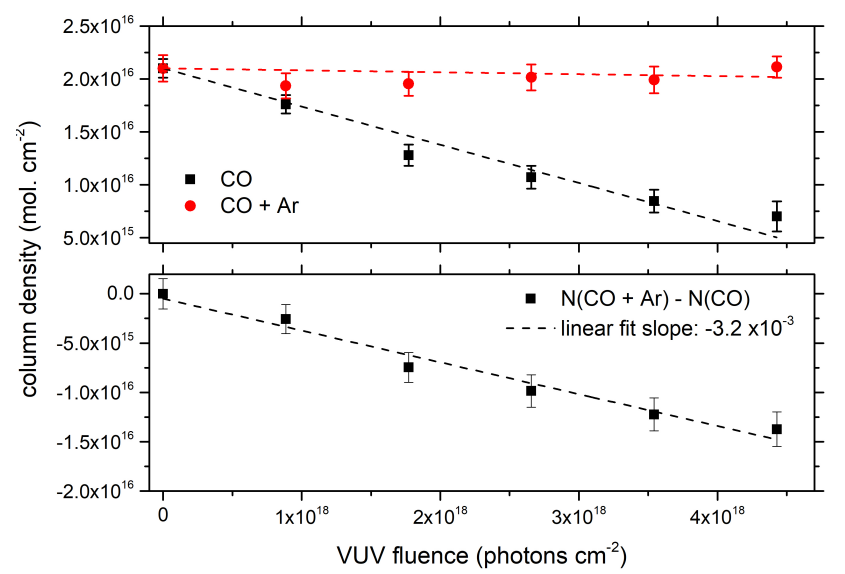

Fig. 5. Upper panel: $\mathrm{CO}$ abundance during the photolysis of $\mathrm{CO}$ with and without the Ar coating at $20 \mathrm{~K}$. Bottom panel: a difference between the $\mathrm{CO}$ and $\mathrm{CO}+\mathrm{Ar}$ experiments resulting in a $\mathrm{CO}$ photodesorption rate.

the ice was irradiated with a fluence of about $4.4 \times 10^{18}$ photons $\mathrm{cm}^{-2}$. Figures 3 and 4 show TOF mass spectra taken at different VUV fluences. For clarity purposes of the plot, we show four out of six spectra taken at different times during the experiment. Figure 3 demonstrates decreasing intensities of peaks at $m / z=12,16$, and 28, which can be directly linked to ongoing photodesorption. In Fig. 4, intensities of $\mathrm{CO}$ peaks stay constant, which means that the Ar layer effectively quenched photodesorption.

In order to calculate the intensity of each peak, a Pearson IV function was fit (Castellanos et al. 2018). A Pearson IV distribution, unlike a Gaussian, allows for peak asymmetry and therefore provides a very good fit for our experimental data. The error bars shown on plots were calculated with the root-mean-square over the residual within one standard deviation from the center of the $\mathrm{m} / \mathrm{z}$ peak.

To derive the photodesorption rate, we calculated the difference in $\mathrm{CO}$ abundance between the two experiments (i.e., $\mathrm{CO}+\mathrm{Ar}$ and $\mathrm{CO}$ ) for every data point and we fit a linear function to obtain the slope as shown in Fig. 5. The analysis yields an average photodesorption rate of $(3.1 \pm 0.3) \times 10^{-3}$ mol. $_{\text {photon }}{ }^{-1}$ in the $7-10.2 \mathrm{eV}$ range, which is in agreement with previous studies. As mentioned in the Introduction, reactions involving photoexcited $\mathrm{CO}$ should be considered. We calculated the 


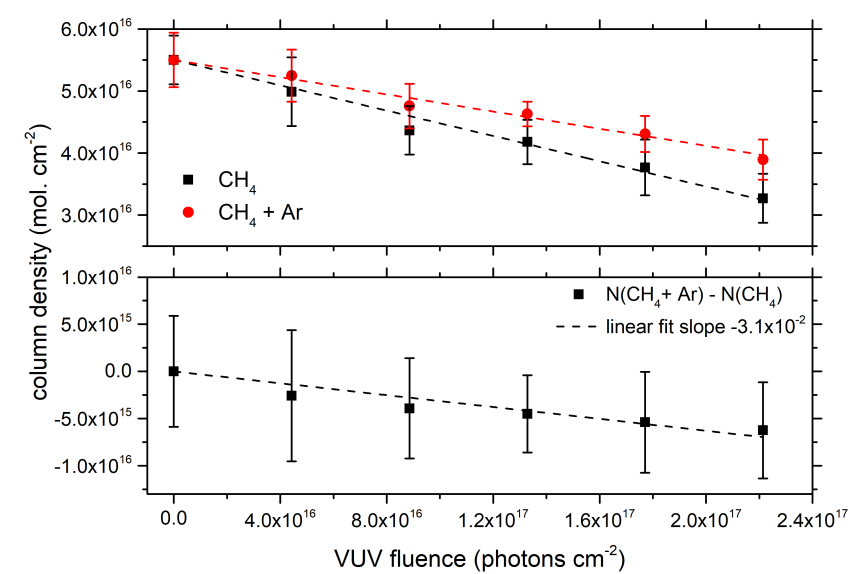

Fig. 6. Upper panel: $\mathrm{CH}_{4}$ abundance during the photolysis of $\mathrm{CH}_{4}$ with and without the Ar coating at $20 \mathrm{~K}$. Bottom panel: a difference between the $\mathrm{CH}_{4}$ and $\mathrm{CH}_{4}+\mathrm{Ar}$ experiments resulting in $\mathrm{CH}_{4}$ photodesorption rate.

efficiency of the $\mathrm{CO}$ to $\mathrm{CO}_{2}$ conversion in our experiments to be at $3.5 \%$ after a fluence of $4.4 \times 10^{18}$ photons $\mathrm{cm}^{-2}$. This constitutes a minor depletion channel for $\mathrm{CO}$ and is in fact within the photodesorption experimental error.

The strength of our method is in comparing the same molecular peaks between two experiments, which eliminates the uncertainty related to calibrating the QMS detector (Martín-Doménech et al. 2015), overlapping IR bands and their absorption coefficients, measuring molecule-dependent pumping speeds, different fragmentation patterns, etc. This illustrates that our photodesorption measurement method works well as it reproduces previous literature values.

\section{Results}

\section{1. $\mathrm{CH}_{4}$}

Solid state $\mathrm{CH}_{4}$ strongly absorbs light around Ly- $\alpha$ with an average cross-section value of $5.7 \times 10^{-18} \mathrm{~cm}^{2}$ for the wavelengths $120-165 \mathrm{~nm}$, while demonstrating almost no absorption at longer wavelengths of the MDHL lamp spectrum (Cruz-Diaz et al. 2014b). The decrease in the methane ice abundance in the photolysis experiments is due to photoconversion (in the bulk and on the surface) as well as photodesorption (only on the surface).

Methane ice of $55 \mathrm{ML}$ was deposited at $20 \mathrm{~K}$ and irradiated by a fluence of up to $2.3 \times 10^{17}$ photons $\mathrm{cm}^{-2}$. In the following experiment, an additional Ar layer of about $50 \mathrm{ML}$ was deposited on top of the $55 \mathrm{ML}$ methane ice and the same experimental procedure was followed. The resulting mass spectra have been analyzed using the procedure described in Sects. 2.3-2.5. The upper panel of Fig. 6 shows integrated mass peaks of methane (contributions from $m / z=12,13,14,15,16$ ) for the two types of experiments. The bottom panel shows the difference between the experiments due to photodesorption. It is fit by a linear function resulting in an average photodesorption yield for methane of $(3.1 \pm 0.5) \times 10^{-2}$ mol. photon ${ }^{-1}$ in the $7-10.2 \mathrm{eV}$ range.

During the analysis of the coated experiment, the unique process that depletes the methane abundance is photoconversion. Given the low total photon fluence, more than $70 \%$ of the original species were left in the ice at the end of the experiment. We applied a linear fit to coated methane photolysis data and derived

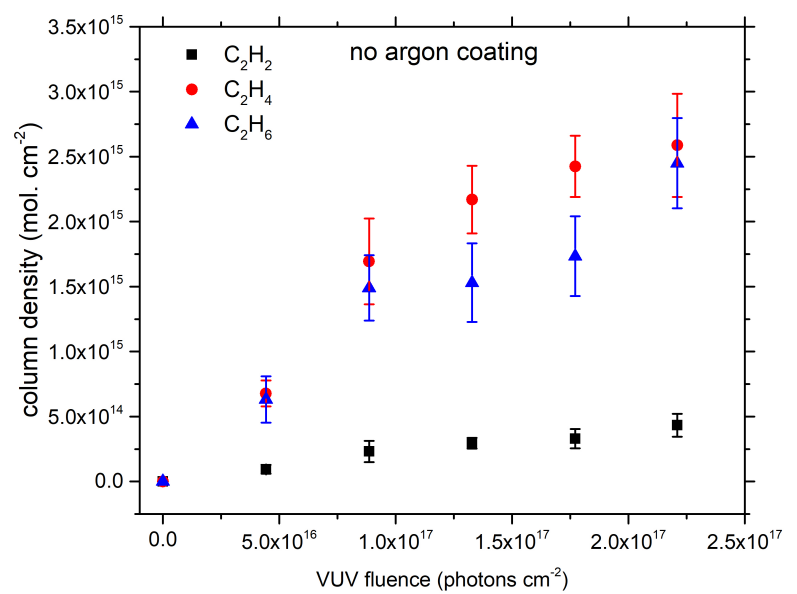

Fig. 7. Obtained $\mathrm{C}_{2} \mathrm{H}_{2}, \mathrm{C}_{2} \mathrm{H}_{4}, \mathrm{C}_{2} \mathrm{H}_{6}$ kinetic curves for photolysis of $\mathrm{CH}_{4}$.

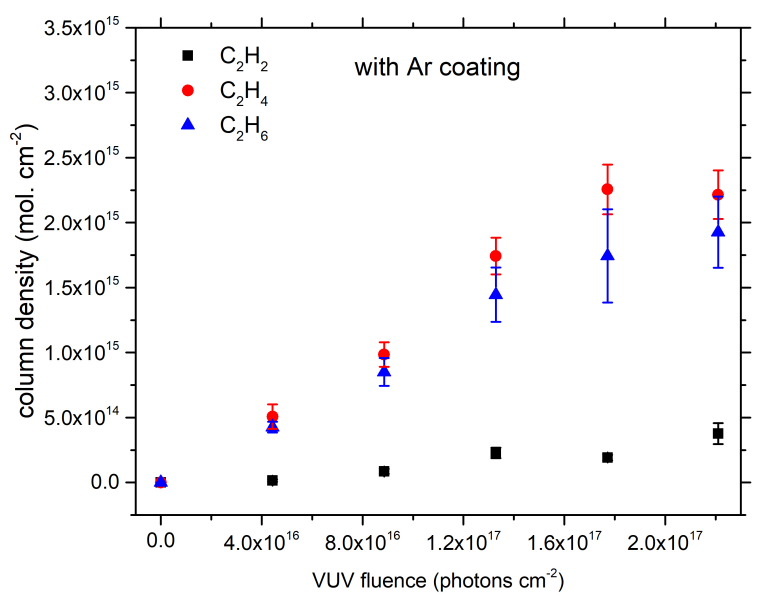

Fig. 8. Obtained $\mathrm{C}_{2} \mathrm{H}_{2}, \mathrm{C}_{2} \mathrm{H}_{4}, \mathrm{C}_{2} \mathrm{H}_{6}$ kinetic curves for photolysis of $\mathrm{CH}_{4}+\mathrm{Ar}$.

an incident photoconversion rate of $7 \times 10^{-2}$ mol. photon ${ }^{-1}$. First order photodissociation products are the following radicals: $\mathrm{CH}$, $\mathrm{CH}_{2}$, and $\mathrm{CH}_{3}$. Given their high reactivity, it is not possible to detect them directly. Nonetheless, we monitored the relative growth of the following second generation photoproducts: acetylene $\left(\mathrm{C}_{2} \mathrm{H}_{2}\right.$, molecular ion $\left.m / z=26\right)$, ethylene $\left(\mathrm{C}_{2} \mathrm{H}_{4}\right.$, $m / z=28)$, and ethane $\left(\mathrm{C}_{2} \mathrm{H}_{6}, m / z=30\right)$. During the derivation of the yields, different electron ionization cross sections at $70 \mathrm{eV}$ and resulting fragmentation patterns were taken into account for $\mathrm{CH}_{4}, \mathrm{C}_{2} \mathrm{H}_{2}, \mathrm{C}_{2} \mathrm{H}_{4}$, and $\mathrm{C}_{2} \mathrm{H}_{6}$ and the ionization cross sections are $3.524,4.374,5.115$, and $6.422 \AA^{2}$, respectively (Kim et al. 2014). Figures 7 and 8 compare the product formation kinetics for both types of experiments. In both experiments, ethylene is the most abundant product, followed closely by ethane, while acetylene is produced in very small amounts. A contribution from fragments of larger products $\left(\mathrm{C}_{3} \mathrm{H}_{y}\right.$ where $\left.y=4,6,8\right)$ toward $\mathrm{C}_{2} \mathrm{H}_{x}(x=2,4,6)$ was very small; therefore, this was not taken into account. Theoretically, a difference in yields of the same photoproduct between (un)coated experiments could be assigned to a combination of dissociative and reactive photodesorption. However, as photoproducts mostly form in the bulk of the ice (90\% of the ice volume), these subtle surface effects are not detected in our data. Additional studies on thin, pure $\mathrm{C}_{2} \mathrm{H}_{2}$, $\mathrm{C}_{2} \mathrm{H}_{4}, \mathrm{C}_{2} \mathrm{H}_{6}$ ices with and without Ar coating are necessary to investigate their intact photodesorption. 


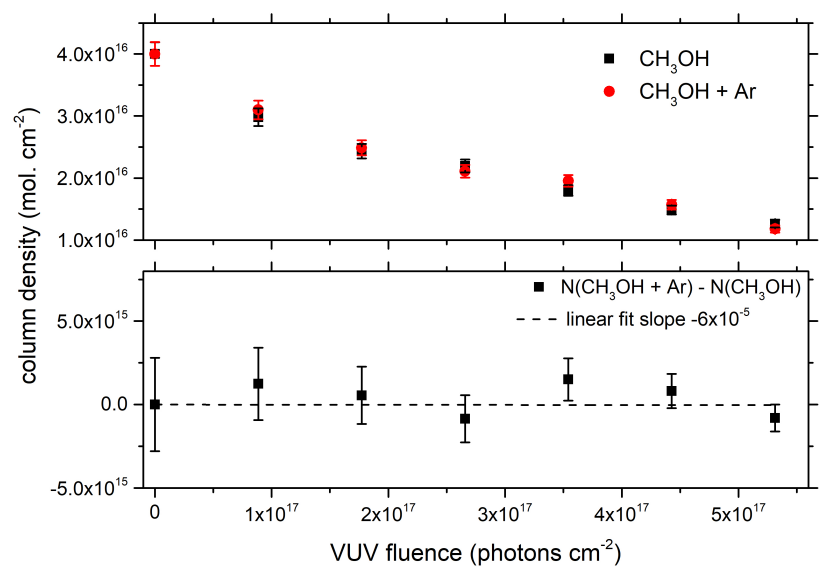

Fig. 9. Upper panel: $\mathrm{CH}_{3} \mathrm{OH}$ abundance during the photolysis of $\mathrm{CH}_{3} \mathrm{OH}$ with and without the $\mathrm{Ar}$ coating at $20 \mathrm{~K}$. Bottom panel: The difference between the $\mathrm{CH}_{3} \mathrm{OH}$ and $\mathrm{CH}_{3} \mathrm{OH}+\mathrm{Ar}$ experiments resulting in a $\mathrm{CH}_{3} \mathrm{OH}$ photodesorption rate. A linear fit is used to constrain an upper limit for $\mathrm{CH}_{3} \mathrm{OH}$ photodesorption rate.

Larger hydrocarbons species were formed in both experiments. It was not our primary focus to investigate the chemical pathways, however it is interesting to note that we recorded signals of large species, containing even up to five carbon atoms (see Fig. 11).

\section{2. $\mathrm{CH}_{3} \mathrm{OH}$}

The solid state $\mathrm{CH}_{3} \mathrm{OH}$ absorption spectrum is a decreasing continuum with an average value of $4.4 \times 10^{-18} \mathrm{~cm}^{2}$ for the wavelengths $120-165 \mathrm{~nm}$ (Cruz-Diaz et al. 2014b). The decrease in the methanol ice abundance in the photolysis experiments is due to photoconversion (in the bulk and on the surface) as well as photodesorption (only on the surface).

Methanol ice of $40 \mathrm{ML}$ was deposited at $20 \mathrm{~K}$ and irradiated by a fluence of $5.3 \times 10^{17}$ photons $\mathrm{cm}^{-2}$. In the following experiment, we added an Ar layer of about 50 ML and applied the same experimental procedure. Integrated signals of methanol remaining in the ice in both experiments are shown in Fig. 9 (combined methanol-only contributions to signals at $m / z=15,29,30,31$, 32). The upper panel demonstrates no visible difference between the coated and uncoated experiments, which suggests photodesorption to be insignificant. The analysis was performed in the same manner as previously described and it results in an upper limit for the desorption efficiency of $<6 \times 10^{-5}$ mol. photon $^{-1}$. Dissociative and reactive photodesorption processes are also below the detection limits. This is in line with recent studies by Bertin et al. (2016) and Cruz-Diaz et al. (2016).

In contrast to photodesorption, the photoconversion of methanol is very efficient. By fitting a linear fit to methanol data, we find a photoconversion rate of $9 \times 10^{-2} \mathrm{~mol}_{\text {. photon }}{ }^{-1}$ and by the end of the experiment, $70 \%$ of the parent species were converted. The subsequent increase in chemical complexity of methanol photoproducts has been studied extensively before (Öberg et al. 2009b; Paardekooper et al. 2016b). Our data also demonstrate the importance of methanol as a precursor of other solid state $\mathrm{COMs}$ in space (e.g., $\mathrm{CH}_{4}, \mathrm{H}_{2} \mathrm{CO}, \mathrm{HCOOCH}_{3}$, $\mathrm{CH}_{3} \mathrm{CHO}$, see Fig. 11).

To confirm that all desorption processes were indeed blocked, we summed up all signals collected by the TOF and tracked the molecular budget during the photolysis, which yields a constant value. We did not take the cross-sections of particular

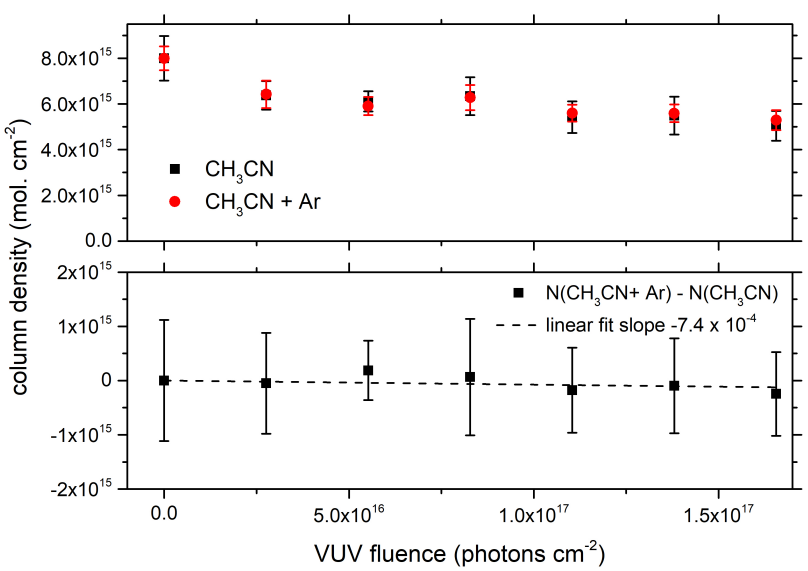

Fig. 10. Upper panel: $\mathrm{CH}_{3} \mathrm{CN}$ abundance during the photolysis of $\mathrm{CH}_{3} \mathrm{CN}$ with and without the $\mathrm{Ar}$ coating at $20 \mathrm{~K}$. Bottom panel: a difference between the $\mathrm{CH}_{3} \mathrm{CN}$ and $\mathrm{CH}_{3} \mathrm{CN}+\mathrm{Ar}$ experiments resulting in a $\mathrm{CH}_{3} \mathrm{CN}$ photodesorption rate. A linear fit is used to constrain an upper limit for $\mathrm{CH}_{3} \mathrm{CN}$ photodesorption rate.

photoproducts into account, but as we compared two experiments with the same products (i.e., same cross sections), this should not affect our conclusion.

\section{3. $\mathrm{CH}_{3} \mathrm{CN}$}

A series of experiments with $8 \mathrm{ML}$ of acetonitrile ice at $20 \mathrm{~K}$ was performed. A layer of about $50 \mathrm{ML}$ of Ar was used to prevent photodesorption processes. Integrated signals of acetonitrile remaining in the ice in both experiments are shown in Fig. 10 (combined acetonitrile-only contributions to signals at $m / z=12$, $13,14,15,25,26,27,28,38,39,41)$. Multiple series of experiments do not demonstrate a detectable level of photodesorption, but this allowed us to determine an upper limit. The linear slope of the difference between capped and uncapped experiments yields $7.4 \times 10^{-4}$ mol. photon ${ }^{-1}$, which we consider to be an upper limit for the photodesorption of acetonitrile.

$\mathrm{CH}_{3} \mathrm{CN}$ experiments were run for a fluence of $1.6 \times$ $10^{17}$ photons $\mathrm{cm}^{-2}$. During the experiment, $35 \%$ of the parent species were photoconverted. The photoconversion rate derived here is $3 \times 10^{-2}$ mol. photon ${ }^{-1}$, which should be considered to be its lower limit since acetonitrile isomerizes to isonitrile and ketenimine, which cannot be separated from the parent species using mass spectrometry (Hudson \& Moore 2004). The efficiencies of these processes are unknown, moreover, these two species are not the only photoproducts. Other products, that we could monitor in our experiments, include $\mathrm{CH}_{4}, \mathrm{HCN} / \mathrm{HNC}$, $\mathrm{CH}_{3} \mathrm{CH}_{2} \mathrm{CN}$, and even larger species (assignments made on the basis of Hudson et al. 2008, see Fig. 11).

\section{Discussion}

\subsection{Comparison with other studies}

Literature values for photodesorption rates of different molecules range between $10^{-1}$ and $10^{-6}$ mol. photon ${ }^{-1}$ (e.g., Chen et al. 2013; Bertin et al. 2016). We first compare our results to previous studies. For CO, our rate $\left((3.1 \pm 0.3) \times 10^{-3}\right.$ mol. photon $\left.^{-1}\right)$ is in agreement with the literature values from experiments as well as theory (e.g., see Paardekooper et al. 2016a,b for an overview of experimental studies, van Hemert et al. 2015 for the theoretical study), proving that our method is reliable for the quantification 
of photodesorption. In the case of methane, intact photodesorption has only been detected by Dupuy et al. (2017), at a rate about an order of magnitude lower than in this work $((2.2 \pm$ $1.1) \times 10^{-3}$ mol. photon $\left.{ }^{-1}\right)$. Several reasons may explain this. The intact photodesorption rate measured by Dupuy et al. accounts for species that desorb from the surface, as a $\mathrm{CH}_{4}$ molecule, through multiple mechanisms (processes 3a, 3b, 3d in Fig. 1). In comparison, the rate derived in our study includes all of the processes mentioned above as well as dissociative desorption (3c). The Ar cap blocks any of the species from leaving the ice surface, including the radicals. In Andersson \& van Dishoeck (2008), dissociative desorption has been shown to be 1-2 orders of magnitude more efficient than in intact desorption (see Table 1 therein). This gives a hint that dissociative desorption can easily account for the difference in the desorption efficiencies between Dupuy et al. (2017) and our study of methane. Additionally, the increased yield derived here may also be related to a higher temperature of the ice: $20 \mathrm{~K}$ in this work, compared to $8-12 \mathrm{~K}$ in the former study. A similar behavior was previously shown for $\mathrm{H}_{2} \mathrm{O}$ and $\mathrm{CO}$ ices (Westley et al. 1995; Arasa et al. 2010; Cruz-Diaz et al. 2018; Öberg et al. 2009a).

As in the case of $\mathrm{CO}$, one order of magnitude deviations are currently considered to be within the experimental acceptance limits. An upper limit derived in the present study for methanol $\left(<6 \times 10^{-5}\right.$ mol. photon $\left.{ }^{-1}\right)$ is in agreement with the other recent studies (Bertin et al. 2016; Cruz-Diaz et al. 2016). The photolysis results indicate that photoconversion into other products is the preferred process following a photon absorption. Methanol fragment photodesorption as well as reactive desorption have been studied before, demonstrating a low efficiency of these processes. The derivation of a photodesorption upper limit of acetonitrile is, to our knowledge, the first quantitative study in the field allowing one to gauge the efficiency of photon triggered transfer of species from a solid state to a gas phase $\left(<7.4 \times 10^{-4}\right.$ mol. photon $\left.{ }^{-1}\right)$. Despite deriving an upper limit, rather than a specific rate, this result can be applied to the state-of-the-art astrochemical models that currently assume a rate in the $10^{-3}$ mol. photon ${ }^{-1}$ range. Similarly to methanol, acetonitrile ice exhibits an efficient photoconversion upon VUV irradiation.

\subsection{Effective yields}

To complete this analysis, an important assumption had to be made regarding the thickness of the ice involved in photodesorption (surface layers). It is based on the findings of previous experimental work investigating the number of $\mathrm{CO}$ monolayers that are actually affected by photodesortion. Bertin et al. (2012) conclude that this process is limited to the upper 2-3 ML. In earlier work by Muñoz Caro et al. (2010), a value of $5 \mathrm{ML}$ is reported. For other molecules, the approximate size of this border region has not been investigated. Here, we therefore assume as a first order approximation that a value of about $3 \mathrm{ML}$ is representative for the molecules investigated in this study $\left(\mathrm{CH}_{4}, \mathrm{CH}_{3} \mathrm{OH}, \mathrm{CH}_{3} \mathrm{CN}\right)$, even though we realize that these species are different in the sense that they also hold the potential to bind through $\mathrm{H}$-bridges. In using the absorption cross section for solid state species from the literature (an average value for the wavelength range $120-165 \mathrm{~nm}$ ), we can determine how many photons are absorbed by a specific ice column density (Sect. 2.4, Eq. (5)). In particular, the top $3 \mathrm{ML}$ of CO ice absorb $\sim 1.5 \%$ of photons, while the bulk and surface combined, $21 \mathrm{ML}$, absorb $10 \%$ of incident photons (absorption cross section of $4.7 \times 10^{-18} \mathrm{~cm}^{2}$ from Cruz-Diaz et al. 2014a,b). The resulting

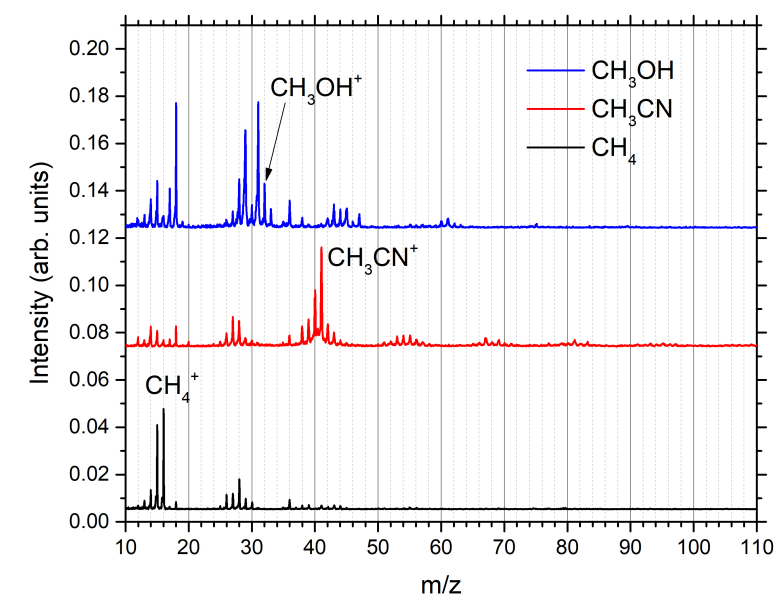

Fig. 11. LDPI TOF MS of $\mathrm{CH}_{4}, \mathrm{CH}_{3} \mathrm{OH}$, and $\mathrm{CH}_{3} \mathrm{CN}$ after a photon fluence of $1.8 \times 10^{17}$ photon $\mathrm{cm}^{-2}$. Peaks at $\mathrm{m} / \mathrm{z}$ higher than the molecular ion represent products created during photoconversion of the species.

effective photodesorption yield of $\mathrm{CO}$ is one desorbed molecule per five photons absorbed in the top three layers $-20 \%$ efficiency. This is in decent agreement with theoretical work by van Hemert et al. (2015), where effective photodesorption yields of amorphous $\mathrm{CO}$ ice at $18 \mathrm{~K}$ was found to be $12.5 \%$ for the surface and subsurface combined (radius of $12.5 \AA \sim 3 \mathrm{ML}$ ).

In the case of methane, by calculating the effective yields, we are able to gauge the competition between the photoconversion, photodesorption, and relaxation. Photoconversion takes place in both the bulk and surface of the ice; we assume that the efficiency of photoconversion for the ice bulk is the same as for the surface layers. We calculated the number of absorbed photons in the top $3 \mathrm{ML}(1.7 \%$ photons) and the whole ice, $55 \mathrm{ML}$ (31\% of incident photons). The derived incident photoconversion rate $(7 \times$ $10^{-2}$ mol. photon ${ }^{-1}$ ) corresponds to an effective yield of 20 converted molecules per 100 absorbed photons; the energy from the remaining 80 absorbed photons is distributed to the ice matrix. When considering the surface layers, a photodesorption rate of $3.1 \times 10^{-2}$ mol. incident photon ${ }^{-1}$ corresponds to 180 depleted methane molecules per 100 absorbed photons. Twenty out of the 180 molecules are photoconverted resulting in 160 photodesorbed methane molecules, per 100 absorbed photons.

A similar analysis can be performed for methanol. We note that $1.4 \%$ of the incident photons are absorbed by the surface layers, while $16 \%$ of photons are absorbed by $40 \mathrm{ML}$ of methanol ice. On average, every 100 photons that are absorbed in the bulk of the ice convert 50 methanol molecules to other species, while the energy from the remaining 50 photons is dissipated in the ice matrix. The effective yield derived in this work is consistent with Cruz-Diaz et al. (2016) where the effective dissociation yield was found to be $0.5 \mathrm{~mol}$. per absorbed photon. The efficiency of photoconversion is more than double compared to $\mathrm{CH}_{4}$. The same photoconversion efficiency applies to the surface layers. However, in order to desorb one methanol molecule, at least 250 photons are required.

The solid state absorption cross section of acetonitrile has not been measured before. Hence, for the purpose of a complete discussion of effective yields, we estimated the solid state absorption cross section of acetonitrile in the following way. Cruz-Diaz et al. (2014a,b) demonstrate that solid state absorption features are broadened and blue shifted with respect to their corresponding gas-phase absorption spectra. Considering that they are related to each other, we calculated the ratio of 
Table 1. Summary of effective yields of photon-triggered processes for investigated solid state species.

\begin{tabular}{llll}
\hline \hline Species & $\begin{array}{l}\text { Photodesorption } \\
\text { yield (mol.) }\end{array}$ & $\begin{array}{l}\text { Photoconversion } \\
\text { yield (mol.) }\end{array}$ & Relaxation (mol.) \\
\hline $\mathrm{CO}$ & 20 & - & 80 \\
& - & - & 100 \\
$\mathrm{CH}_{4}$ & 160 & 20 & 80 \\
& - & 20 & 80 \\
$\mathrm{CH}_{3} \mathrm{OH}$ & $<0.04$ & 50 & 50 \\
& - & 50 & 50 \\
$\mathrm{CH}_{3} \mathrm{CN}$ & $<0.3$ & 50 & 50 \\
& - & 50 & 50 \\
\hline
\end{tabular}

Notes. Yields are given in molecules per 100 absorbed photons. First row for each molecule corresponds to processes in the active surface layers ( $3 \mathrm{ML}$ ), while the second row represents the ice bulk. The uncertainty for these values is estimated to be $\pm 10 \%$.

the gas phase to the solid state absorption cross section for methanol $\left(8.2 \times 10^{-18} \mathrm{~cm}^{2}\right.$ from Salahub \& Sandorfy 1971 and $4.4 \times 10^{-18} \mathrm{~cm}^{2}$ from Cruz-Diaz et al. 2014a,b, respectively) and applied it to calculate the cross section of acetonitrile in the solid state from its average gas phase absorption cross section $\left(1.4 \times 10^{-17} \mathrm{~cm}^{2}\right.$ from Suto \& Lee 1985$)$. This simplified calculation yields an average absorption cross section of $7.5 \times 10^{-18} \mathrm{~cm}^{2}$ for solid state $\mathrm{CH}_{3} \mathrm{CN}$ in the range $120-165 \mathrm{~nm}$. Taking into account the estimated cross section, the top $3 \mathrm{ML}$ absorb $2 \%$ of the incident photons, while $8 \mathrm{ML}$ of the ice absorb $6 \%$ of the incident photons. Thus, upon the absorption of 100 photons, 50 molecules dissociate and form other species with the same efficiency in the bulk and surface layers. The upper limit of photodesorption is converted to one desorbed molecule per at least 30 absorbed photons. The summary of the calculated effective yields is presented in Table 1, normalized to 100 photons.

\subsection{Photodesorption mechanisms}

The total absorption cross section for each of the studied molecules is of the same order of magnitude, which excludes it as the key factor that determines the competition between photodesorption and photoconversion. In an attempt to explain the difference in orders of magnitude in photodesorption rates for the molecules studied here, one needs to investigate the different mechanisms driving this process and its competition with photoconversion. The present study, with a wider scope thanks to results from other work, allows one to link the physical parameters to the possible mechanism involved.

For CO, desorption induced by electronic transitions (DIET) is an indirect process responsible for photodesorption below $10 \mathrm{eV}$ range. Electronic excitation energy that is redistributed to neighbors provides energy to surface molecules in order to overcome (van der Waals type) bond energies that bind them to the ice (Fayolle et al. 2011). Photodissociation and ionization of CO are both negligible processes.

The photodesorption mechanism for dissociative species (e.g., methane, methanol, acetonitrile) is not fully understood. It is likely that different mechanisms are at play, either simultaneously or sequentially. For the molecules studied here, photodissociation can occur through the loss of a single hydrogen atom, which can carry energy to a surface molecule leading to its desorption. This $\mathrm{H}$ atom kick-out mechanism has previously been proposed by molecular dynamics (MD) simulations
Table 2. Summary of parameters that impact the efficiency of the kickout photodesorption mechanism.

\begin{tabular}{lllll}
\hline \hline Species & $\begin{array}{l}\text { Intermol. } \\
\text { bond } \\
\text { energy }(\mathrm{eV})\end{array}$ & $\begin{array}{l}\text { Diss. } \\
\text { threshold } \\
\text { energy }(\mathrm{eV})\end{array}$ & $\begin{array}{l}\text { Energy trans. } \\
\text { to a surface } \\
\text { species }(\mathrm{eV})\end{array}$ & Ratio \\
\hline $\mathrm{CH}_{4}$ & 0.1 & 4.5 & $<0.99$ & 9.9 \\
$\mathrm{CH}_{3} \mathrm{OH}$ & 0.38 & 4.5 & $<0.58$ & 1.5 \\
$\mathrm{CH}_{3} \mathrm{CN}$ & 0.39 & 4 & $<0.46$ & 1.2 \\
\hline
\end{tabular}

Notes. The last column is the ratio of the upper limit for energy transferred to surface species to the intermolecular bond energy (see text below for details).

of water ice (Andersson \& van Dishoeck 2008) and may more generally apply as is discussed below.

If we make the assumption that the $\mathrm{H}$ atom does not lose any energy in the ice until it collides with a surface molecule, this can lead to desorption given that the intermolecular bond energy is overcome. The initial energy of the $\mathrm{H}$ atom is determined by the remaining energy after dissociation. In our calculations, we assumed that all energy goes into the kinetic energy of the $\mathrm{H}$ atom. Following the laws of conservation of energy and momentum, we calculated the upper limit of the energy transferred to the surface molecule. Comparing this with the intermolecular bond strength gives a hint about the efficiency of the kick-out desorption mechanism by $\mathrm{H}$ atoms. The results of these calculations are summarized in Table 2, and they are discussed in detail in the following paragraphs.

The dissociation of condensed methane is initiated by photon absorption (average energy of $9 \mathrm{eV}$ ), and subsequently the $\mathrm{H}-\mathrm{CH}_{3}$ bond is broken (BDE of $4.5 \mathrm{eV}$ ). We assume that the $\mathrm{H}$ atom inherits all remaining energy in the form of kinetic energy $(4.5 \mathrm{eV})$. The energetic fragment moves through the ice and collides with a surface molecule to transfer up to $0.99 \mathrm{eV}$. This value exceeds the energy required to break the intermolecular bond of methane $(0.1 \mathrm{eV}$, Smith et al. 2015) with a factor of 10 . This particularly high ratio might help to account for the effective photodesorption rate of methane (see Table 1).

The photodissociation of methanol liberates the $\mathrm{H}$ atom through breaking the $\mathrm{H}-\mathrm{CH}_{2} \mathrm{OH}$ bond $(4.16 \mathrm{eV})$ or $\mathrm{CH}_{3} \mathrm{O}-\mathrm{H}$ (4.5 eV) (Blanksby \& Ellison 2003). The same calculation procedure as for methane yields an upper limit of the energy transferred to the surface molecule of $0.58 \mathrm{eV}$. A comparison with the strong hydrogen bonding energy of the methanol ice $(0.38 \mathrm{eV}$, Martín-Doménech et al. 2014) yields a ratio of 1.5. This low ratio can indicate that kick-out desorption is a possible mechanism for methanol, but given our idealistic assumptions, it is unlikely to be efficient. The case of acetonitrile is similar to methanol. The photodissociation threshold of $\mathrm{CH}_{3} \mathrm{CN}$ was set by the $\mathrm{BDE}$ of $\mathrm{H}-\mathrm{CH}_{2} \mathrm{CN}$ bond at $4.01 \mathrm{eV}$ (Schwell et al. 2008). The $\mathrm{H}$ atom can transfer up to $0.46 \mathrm{eV}$ of energy to a strongly bound surface molecule (intermolecular bonding energy of $0.39 \mathrm{eV}$, Abdulgalil et al. 2013). The ratio of the upper limit for transferred energy to the intermolecular bonding is 1.2. In an ideal case, this should be sufficient for desorption, but in reality the $\mathrm{H}$ atom loses some energy when traveling through the ice before colliding with the surface molecule. Hence, this mechanism is expected to be less efficient.

If we consider the kick-out mechanism to be a dominant photodesorption process for dissociative species, our data suggest a trend related to their efficiency. In particular, the photodesorption rate can be roughly estimated by comparing the 
upper limit for the energy transferred to the surface via perfect collision and intermolecular bonding energy. This ratio can possibly be linked to the measured photodesorption rate. If this trend holds true, then under the made assumptions, photodesorption of larger molecules, that are strongly bound, likely fall below $10^{-3}$ mol. photon ${ }^{-1}$, the current default astrochemical model value.

The efficiency of the kick-out process has been discussed in the context of pure ices, but more importantly, it is likely that this process also plays a role in the interstellar medium. If this is the case, our results can be extrapolated toward realistic conditions. For example if we look at the water-rich phase of interstellar ices. These ices contain minor abundances of $\mathrm{CH}_{4}, \mathrm{CO}_{2}$, and $\mathrm{NH}_{3}$ (Garrod \& Pauly 2011), which are likely to be isolated in a water matrix or on a surface bound to the water ice. The kick-out photodesorption efficiency of these species can be determined by the comparison of energy binding them to the water ice (for overview see Table 2 in Das et al. 2018) and the energy which can be transferred to these species by an intermediate carrier, such as an $\mathrm{H}$ atom. We can then follow the same procedure as in Table 2. We assume that the $\mathrm{H}$ atom is created upon water dissociation and can transfer its energy to a surface $\mathrm{CH}_{4}, \mathrm{CO}_{2}$, or $\mathrm{NH}_{3}$ molecule. The resulting ratios suggest that the kick-out mechanism is the most efficient for methane (ratio of 10.3), then $\mathrm{CO}_{2}$ (1.8) and $\mathrm{NH}_{3}$ (1.7). To complete these calculations, the following binding energies were used: $0.08 \mathrm{eV}$ (Raut et al. 2007), $0.2 \mathrm{eV}$ (Minissale et al. 2016), and 0.48 eV (Collings et al. 2004), respectively.

It is important to mention that the kick-out mechanism is not the only mechanism capable of photodesorption. Alternatively, for a surface methane molecule, the fragments $\mathrm{CH}_{3}$ and $\mathrm{H}$ can recombine following an exothermic reaction with $4.5 \mathrm{eV}$ excess energy. Upon comparison with the intermolecular bond energy, this is sufficient to desorb from the surface (Chase 1998). Recombination reactions of gas phase radicals, which are formed in this way, have been proposed as a starting point for a gas phase formation route to $\mathrm{CH}_{3} \mathrm{OH}$ (Bertin et al. 2016).

Theoretical studies, such as for CO (van Hemert et al. 2015) and $\mathrm{H}_{2} \mathrm{O}$ (Andersson \& van Dishoeck 2008), are essential for investigating the mechanisms behind VUV photodesorption. Hence, molecular dynamics studies of methane, methanol, and acetonitrile are necessary to confirm or disprove our hypothesis.

\subsection{Astrophysical implications}

As mentioned in the Introduction, photodesorption has been considered to be an effective way to transfer, newly formed, solid state species into the gas phase. In the case of the nondissociative VUV excitation of CO, this seems to apply; the measured photodesorption rates, presented here and in previous studies, are high enough to explain $\mathrm{CO}$ gas phase observations toward the prestellar core B68 (Bergin et al. 2006). This result is even more relevant, since icy dust grains are expected to be coated with a CO-rich layer, as $\mathrm{CO}$ is the last abundant molecule to freeze out. Determining the relevance of photodesorption as a mechanism that bridges the solid state and gas phase becomes much more challenging when looking for other species, such as $\mathrm{H}_{2} \mathrm{O}$ or $\mathrm{CO}_{2}$, which easily dissociate upon VUV excitation. The new technique presented here has an advantage that it can be used to study several photoprocesses taking place simultaneously (i.e., photodesorption and photoconversion). Due to this, the method has been applied to stable $\mathrm{CO}$, dissociating $\mathrm{CH}_{4}$, and $\mathrm{CH}_{3} \mathrm{OH}$ ices, largely confirming data from previous work. This confirmation is of particular interest for $\mathrm{CH}_{3} \mathrm{OH}$, since this study also shows that it is very hard to photodesorb intact methanol. Additionally, dissociative desorption may be an important mechanism, as suggested for the case of $\mathrm{CH}_{4}$. It may be very well possible that the resulting radicals recombine in the gas phase, that is, reflecting a solid state triggered gas phase chemistry. For the dissociative VUV irradiation of $\mathrm{CH}_{3} \mathrm{CN}$, no earlier photodesorption values have been presented and an upper limit has been derived here that is about a factor 10 lower than the values typically used in astrochemical models. There is a second advantage regarding the new method. The existing techniques have focused on pure ices. The only exceptions concern studies focusing on codesorption or reactive desorption experiments, such as for mixed $\mathrm{CO}: \mathrm{N}_{2}, \mathrm{CO}: \mathrm{CH}_{3} \mathrm{OH}$, and $\mathrm{CH}_{4}: \mathrm{H}_{2} \mathrm{O}$ ices (Fayolle et al. 2013; Bertin et al. 2013; Martín-Doménech et al. 2016). The LDPI TOF MS method discussed here is also capable of dealing with mixed, that is, astronomically more realistic ices. It will be interesting to know how different ice constituents determine each other's photodesorption properties, as a function of the mixing ratio, but also, for example, for different temperatures. This is currently a work in progress. From the work presented here, it is already clear that photoconversion increases the number of ice constituents substantially. This is illustrated in Fig. 11. The photoconversion not only decreases the abundance of precursor material, but it also increases the molecular complexity in the ice. The figure shows the TOF mass spectra for methanol, acetonitrile, and methane ices upon VUV irradiation for an irradiation of only $12 \mathrm{~min}$. As discussed earlier (Öberg et al. 2009b; Paardekooper et al. 2016b), larger COMs are formed in this process as well. It is clear that photodesorption and photoconversion are two intimately mixed processes.

From the present work, another astronomically relevant conclusion can be drawn. The coating on top of the ices prohibits surface radicals, which normally desorb, to leave the ice. It is a logical step to assume that these radicals are, therefore, forced to recombine in the ice, enhancing the overall photoconversion. Such a process could take place in space as well, as interstellar ices are shown to have layered structures, that is, the $\mathrm{CO}$-coating on top of a $\mathrm{H}_{2} \mathrm{O}$ rich ice could enforce chemistry involving $\mathrm{OH}$ radicals. Here, the impact of the coating as a reaction thriving mechanism could not be confirmed.

To summarize, the present work on pure ices, comprising of molecules dissociating upon VUV irradiation, cannot explain the observed gas phase abundances of methanol and acetonitrile. It is very likely, that for even larger complex organic molecules, the intact desorption efficiency will go even further down. Nevertheless, the detection of such species on cometary surfaces as well as the proof for their thermal release from icy dust grains (Lee et al. 2019) clearly hints at a solid state origin for these species. This is also fully in line with the data presented here, see Fig. 11. A possible explanation for this inconsistency is that most studies have focused on pure ices so far and the processes in mixed ices may be different (e.g., efficient reactive desorption). The new method that is introduced here holds the potential to study these processes further; also it provides a versatile tool that is able to discriminate between direct photodesorption and photoconversion.

\section{Conclusions}

We introduce a new method for gauging the competition between photon-triggered processes in solid state species. By comparing time-of-flight mass spectra of laser ablated (un)coated ice experiments upon VUV irradiation, we are able to distinguish between 
Table 3. Summary of photon-triggered processes of solid state species at $20 \mathrm{~K}$ in the $7-10.2 \mathrm{eV}$ photon energy range.

\begin{tabular}{lll}
\hline \hline Species & $\begin{array}{l}\text { Photodesorption } \\
\text { rate } \\
\text { (in mol. photon }\end{array}$ & $\begin{array}{l}\text { Photoconvertion } \\
\text { cross section (in } \\
\mathrm{cm}^{2} \text { ) }\end{array}$ \\
\hline $\mathrm{CO}$ & $(3.1 \pm 0.3) \times 10^{-3}$ & - \\
$\mathrm{CH}_{4}$ & $(3.1 \pm 0.5) \times 10^{-2}$ & $3.7 \times 10^{-18}$ \\
$\mathrm{CH}_{3} \mathrm{OH}$ & $<6.0 \times 10^{-5}$ & $2.8 \times 10^{-18}$ \\
$\mathrm{CH}_{3} \mathrm{CN}$ & $<7.4 \times 10^{-4}$ & $3.0 \times 10^{-17}$ \\
\hline
\end{tabular}

the effects of photodesorption and photoconversion. The new approach has been demonstrated on the example of $\mathrm{CO}$, and then applied to $\mathrm{CH}_{4}, \mathrm{CH}_{3} \mathrm{OH}$, and $\mathrm{CH}_{3} \mathrm{CN}$. The derived rates are average values in the $7-10.2 \mathrm{eV}$ photon energy range and are shown in Table 3.

For $\mathrm{CH}_{3} \mathrm{CN}$, for the first time, a value for the photodesorption rate is presented that provides an upper limit that is about a factor of 10 lower than what is currently used in astrochemical models. Moreover, upon comparison of the values determined for pure species studied here and in some earlier work, the following trend is suggested: the VUV desorption efficiency for dissociative species seems to depend on the balance between the extra energy available after dissociation, intermolecular bonding energy, and the relative mass of fragments and parent species. This implies that for even larger complex organic molecules (compared to $\mathrm{CH}_{3} \mathrm{OH}$ and $\mathrm{CH}_{3} \mathrm{CN}$ ), the intact photodesorption rate likely falls below the currently used rate of $10^{-3}$ mol. photon ${ }^{-1}$. It should be noted that this behavior has been observed in laboratory experiments containing pure ices, with a few exceptions in binary mixtures (Bertin et al. 2016; Zhen \& Linnartz 2014); whereas, in the ISM, ices are generally mixed. Since the new technique also allows one to study realistic ices analogs, future studies will help in quantitatively disentangling the role that impacting VUV photons play in interstellar chemistry.

Acknowledgements. $\mathrm{MB}$ and HL acknowledges the European Union (EU) and Horizon 2020 funding awarded under the Marie Skłodowska-Curie action to the EUROPAH consortium (grant number 722346) as well as NOVA 5 funding. DMP thanks NASA Postdoctoral program for a NPP fellowship. We thank N. Ligterink and A. G. G. M. Tielens for helpful discussions.

\section{References}

Abdulgalil, A. G. M., Marchione, D., Thrower, J. D., et al. 2013, Philos. Trans. R. Soc. A, 371, 20110586

Abplanalp, M. J., \& Kaiser, R. I. 2019, Phys. Chem. Chem. Phys. (Incorp. Far. Trans.), 21, 16949

Andersson, S., \& van Dishoeck, E. F. 2008, A\&A, 491, 907

Arasa, C., Andersson, S., Cuppen, H. M., van Dishoeck, E. F., \& Kroes, G. J. 2010, J. Chem. Phys., 132, 184510

Bergin, E. A., Maret, S., van der Tak, F. F. S., et al. 2006, ApJ, 645, 369

Bergner, J. B., Guzmán, V. G., Öberg, K. I., Loomis, R. A., \& Pegues, J. 2018, ApJ, 857, 69

Bertin, M., Fayolle, E. C., Romanzin, C., et al. 2012, Phys. Chem. Chem. Phys. (Incorp. Far. Trans.), 14, 9929

Bertin, M., Fayolle, E. C., Romanzin, C., et al. 2013, ApJ, 779, 120

Bertin, M., Romanzin, C., Doronin, M., et al. 2016, ApJ, 817, L12

Blanksby, S. J., \& Ellison, G. B. 2003, Acc. Chem. Res., 36, 255

Boamah, M. D., Sullivan, K. K., Shulenberger, K. E., et al. 2014, Far. Disc., 168 , 249

Boogert, A. C. A., Huard, T. L., Cook, A. M., et al. 2011, ApJ, 729, 92

Boogert, A. C. A., Gerakines, P. A., \& Whittet, D. C. B. 2015, ARA\&A, 53, 541
Bossa, J. B., Paardekooper, D. M., Isokoski, K., \& Linnartz, H. 2015, Phys. Chem. Chem. Phys. (Incorp. Far. Trans.), 17, 17346

Brunetto, R., Caniglia, G., Baratta, G., \& Palumbo, M. 2008, ApJ, 686, 1480

Calcutt, H., Jørgensen, J. K., Müller, H. S. P., et al. 2018, A\&A, 616, A90

Castellanos, P., Candian, A., Zhen, J., Linnartz, H., \& Tielens, A. G. G. M. 2018, A\&A, 616, A166

Cernicharo, J., Marcelino, N., Roueff, E., et al. 2012, ApJ, 759, L43

Chase, Jr., M. W. 1998, Journal of Physical and Chemical Reference Data (New York: American Institute of Physics)

Chen, Y.-J., Chuang, K.-J., Caro, G. M., et al. 2013, ApJ, 781, 15

Chiar, J. E., Adamson, A. J., \& Whittet, D. C. B. 1996, ApJ, 472, 665

Chuang, K. J., Fedoseev, G., Qasim, D., et al. 2017, MNRAS, 467, 2552

Chuang, K. J., Fedoseev, G., Qasim, D., et al. 2018, ApJ, 853, 102

Collings, M. P., Anderson, M. A., Chen, R., et al. 2004, MNRAS, 354, 1133

Cordiner, M. A., Palmer, M. Y., Nixon, C. A., et al. 2015, ApJ, 800, L14

Cruz-Diaz, G., Caro, G. M., Chen, Y.-J., \& Yih, T.-S. 2014a, A\&A, 562, A119

Cruz-Diaz, G. A., Muñoz Caro, G. M., Chen, Y. J., \& Yih, T. S. 2014b, A\&A, 562, A 120

Cruz-Diaz, G. A., Martín-Doménech, R., Muñoz Caro, G. M., \& Chen, Y. J. 2016, A\&A, 592, A68

Cruz-Diaz, G. A., Martín-Doménech, R., Moreno, E., Muñoz Caro, G. M., \& Chen, Y.-J. 2018, MNRAS, 474, 3080

Cuppen, H. M., \& Herbst, E. 2007, ApJ, 668, 294

Dartois, E., Augé, B., Boduch, P., et al. 2015, A\&A, 576, A125

Das, A., Sil, M., Gorai, P., Chakrabarti, S. I. K., \& Loison, J. C. 2018, ApJS, 237, 9

Dobbs, E. R., Figgins, B. F., Jones, G. O., Piercey, D. C., \& Riley, D. P. 1956, Nature, 178, 483

Dupuy, R., Bertin, M., Féraud, G., et al. 2017, A\&A, 603, A61

Dupuy, R., Bertin, M., Féraud, G., et al. 2018, Nat. Astron., 2, 796

Fayolle, E. C., Bertin, M., Romanzin, C., et al. 2011, ApJ, 739, L36

Fayolle, E. C., Bertin, M., Romanzin, C., et al. 2013, A\&A, 556, A122

Fedoseev, G., Cuppen, H. M., Ioppolo, S., Lamberts, T., \& Linnartz, H. 2015, MNRAS, 448, 1288

Fillion, J. H., Fayolle, E. C., Michaut, X., et al. 2014, Faraday Discuss., 168, 533

Fuchs, G. W., Cuppen, H. M., Ioppolo, S., et al. 2009, A\&A, 505, 629

Gans, B., Peng, Z., Carrasco, N., et al. 2013, Icarus, 223, 330

Garrod, R. T., \& Pauly, T. 2011, ApJ, 735, 15

Garrod, R. T., Widicus Weaver, S. L., \& Herbst, E. 2008, ApJ, 682, 283

Gerakines, P. A., Schutte, W. A., \& Ehrenfreund, P. 1996, A\&A, 312, 289

Goesmann, F., Rosenbauer, H., Bredehöft, J. H., et al. 2015, Science, 349, 2689

Guzmán, V. V., Goicoechea, J. R., Pety, J., et al. 2013, A\&A, 560, A73

Henderson, B. L., \& Gudipati, M. S. 2015, ApJ, 800, 66

Herbst, E., \& van Dishoeck, E. F. 2009, ARA\&A, 47, 427

Hudgins, D. M., Sandford, S. A., Allamandola, L. J., \& Tielens, A. G. G. M. 1993, ApJS, 86, 713

Hudson, R. L., \& Moore, M. H. 2004, Icarus, 172, 466

Hudson, R. L., Moore, M. H., Dworkin, J. P., Martin, M. P., \& Pozun, Z. D. 2008, Astrobiology, 8, 771

Kalescky, R., Kraka, E., \& Cremer, D. 2013, J. Phys. Chem. A, 117, 8981

Kalvāns, J. 2015, ApJ, 803, 52

Kim, Y. K., Irikura, K. K., Rudd, M. E., \& Ali, M. A. 2014, Electron-Impact Ionization Cross Section for Ionization and Excitation Database (version 3.0)

Kofman, V., He, J., Loes ten Kate, I., \& Linnartz, H. 2019, ApJ, 875, 131

Lee, J.-E., Lee, S., Baek, G., et al. 2019, Nat. Astron., 3, 314

Ligterink, N. F. W., Paardekooper, D. M., Chuang, K. J., et al. 2015, A\&A, 584, A56

Ligterink, N. F. W., Walsh, C., Bhuin, R. G., et al. 2018, A\&A, 612, A88

Lo, J.-I., Lin, M.-Y., Peng, Y.-C., et al. 2015, MNRAS, 451, 159

Luna, R., Molpeceres, G., Ortigoso, J., et al. 2018, A\&A, 617, A116

Maity, S., Kaiser, R. I., \& Jones, B. M. 2014, Far. Disc., 168, 485

Martín-Doménech, R., Muñoz Caro, G. M., Bueno, J., \& Goesmann, F. 2014, A\&A, 564, A8

Martín-Doménech, R., Manzano-Santamaría, J., Muñoz Caro, G. M., et al. 2015, A\&A, 584, A14

Martín-Doménech, R., Muñoz Caro, G. M., \& Cruz-Díaz, G. A. 2016, A\&A, 589, A107

Mathis, J. S., Mezger, P. G., \& Panagia, N. 1983, A\&A, 500, 259

Minissale, M., Dulieu, F., Cazaux, S., \& Hocuk, S. 2016, A\&A, 585, A24

Muñoz Caro, G. M., Jiménez-Escobar, A., Martín-Gago, J. Á., et al. 2010, A\&A, 522, A108

Oba, Y., Tomaru, T., Lamberts, T., Kouchi, A., \& Watanabe, N. 2018, Nat. Astron., 2, 228

Öberg, K. I., van Dishoeck, E. F., \& Linnartz, H. 2009a, A\&A, 496, 281 
Öberg, K. I., Garrod, R. T., van Dishoeck, E. F., \& Linnartz, H. 2009b, A\&A, 504,891

Öberg, K. I., Furuya, K., Loomis, R., et al. 2015, ApJ, 810, 112

Paardekooper, D. M., Bossa, J. B., Isokoski, K., \& Linnartz, H. 2014, Rev. Sci. Instr., 85, 104501

Paardekooper, D. M., Bossa, J. B., \& Linnartz, H. 2016a, A\&A, 592, A67

Paardekooper, D. M., Fedoseev, G., Riedo, A., \& Linnartz, H. 2016b, A\&A, 596, A72

Purcell, C. R., Balasubramanyam, R., Burton, M. G., et al. 2006, MNRAS, 367 553

Raut, U., Teolis, B. D., Loeffler, M. J., et al. 2007, J. Chem. Phys., 126, 244511

Riddick, J. A., Bunger, W. B., \& Sakano, T. K. 1986, Organic Solvents: Physica Properties and Methods of Purification (New York, NY: John Wiley and Sons)

Roux, J. A., Wood, B. E., Smith, A. M., \& Plyer, R. R. 1980, Final Report (ARO Inc, Arnold Air Force Station, TN)

Ruscic, B. 2015, J. Phys. Chem. A, 119, 7810

Salahub, D. R., \& Sandorfy, C. 1971, Chem. Phys. Lett., 8, 71

Schnepp, O., \& Dressler, K. 1960, J. Chem. Phys., 33, 49

Schwell, M., Jochims, H.-W., Baumgärtel, H., \& Leach, S. 2008, Chem. Phys., 344,164
Sinnock, A. C., \& Smith, B. L. 1968, Phys. Lett. A, 28, 22

Smith, R. S., May, R. A., \& Kay, B. D. 2015, J. Phys. Chem. A B, 120, 1979

Solomon, P. M., Jefferts, K. B., Penzias, A. A., \& Wilson, R. W. 1971, ApJ, 168, L107

Suto, M., \& Lee, L. C. 1985, J. Geophys. Res., 90, 037

van Hemert, M. C., Takahashi, J., \& van Dishoeck, E. F. 2015, J. Phys. Chem. A, 119,6354

Vastel, C., Ceccarelli, C., Lefloch, B., \& Bachiller, R. 2014, ApJ, 795, L2

Walsh, C., Herbst, E., Nomura, H., Millar, T. J., \& Weaver, S. W. 2014, Far Disc., 168, 389

Watanabe, N., \& Kouchi, A. 2002, ApJ, 571, L173

Weast, R. C. 1972, Handbook of Chemistry and Physics, 53rd ed. (The Chemical Rubber Co.), 26

Westley, M. S., Baragiola, R. A., Johnson, R. E., \& Baratta, G. A. 1995, Planet. Space Sci., 43, 1311

Willacy, K., \& Langer, W. D. 2000, ApJ, 544, 903

Woodney, L. M., A'Hearn, M. F., Schleicher, D. G., et al. 2002, Icarus, 157, 193

Zhen, J., \& Linnartz, H. 2014, MNRAS, 437, 3190 